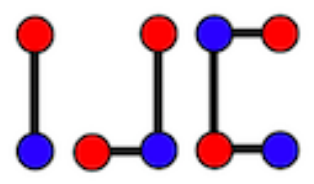

\title{
Computing total edge irregularity strength of some $n$-uniform cactus chain graphs and related chain graphs
}

\author{
Isnaini Rosyida ${ }^{\mathrm{a}}$, Diari Indriati ${ }^{\mathrm{b}}$ \\ ${ }^{a}$ Department of Mathematics, Faculty of Mathematics and Natural Sciences, \\ Universitas Negeri Semarang, Indonesia. \\ ${ }^{b}$ Departments of Mathematics, Faculty of Mathematics and Natural Sciences, \\ Universitas Sebelas Maret, Surakarta, Indonesia \\ iisisnaini@gmail.com,diari_indri@yahoo.co.id
}

\begin{abstract}
Given graph $G(V, E)$. We use the notion of total $k$-labeling which is edge irregular. The notion of total edge irregularity strength (tes) of graph $G$ means the minimum integer $k$ used in the edge irregular total $k$-labeling of $G$. A cactus graph $G$ is a connected graph where no edge lies in more than one cycle. A cactus graph consisting of some blocks where each block is cycle $C_{n}$ with same size $n$ is named an $n$-uniform cactus graph. If each cycle of the cactus graph has no more than two cut-vertices and each cut-vertex is shared by exactly two cycles, then $G$ is called $n$-uniform cactus chain graph. In this paper, we determine tes of $n$-uniform cactus chain graphs $C\left(C_{n}^{r}\right)$ of length $r$ for some $n \equiv 0 \bmod 3$. We also investigate tes of related chain graphs, i.e. tadpole chain graphs $T_{r}(4, n)$ and $T_{r}(5, n)$ of length $r$. Our results are as follows: $\operatorname{tes}\left(C\left(C_{n}^{r}\right)\right)=\left\lceil\frac{n r+2}{3}\right\rceil$; $\operatorname{tes}\left(T_{r}(4, n)\right)=\left\lceil\frac{(4+n) r+2}{3}\right\rceil ;$ and tes $\left(T_{r}(5, n)\right)=\left\lceil\frac{(5+n) r+2}{3}\right\rceil$.
\end{abstract}

Keywords: edge irregular total $k$-labeling, total edge irregularity strength, uniform, cactus chain, tadpole graph. Mathematics Subject Classification: 05C78

DOI: $10.19184 /$ ijc.2020.4.1.6

Received: 5 June 2019, Revised: 14 April 2020, Accepted: 27 May 2020. 


\section{Introduction}

Let $G=(V, E)$ be a finite, undirected, and simple graph. An edge irregular total $k$-labeling of $G$ is a function $f: V \cup E \rightarrow\{1,2, \ldots, k\}$ that satisfies the weight $w t(u v) \neq w t(x y)$ for every two different edges $u v$ and $x y$ in $E(G)$ with $w t(u v)=f(u)+f(v)+f(u v)$ [4]. The minimum integer $k$ in total labeling $f$ is called the total edge irregularity strength of $G$, symbolized by tes $(G)$. Further, Bača et al. ([4], [5]) also provided the lower and upper bounds:

$$
\left\lceil\frac{|E(G)|+2}{3}\right\rceil \leq \operatorname{tes}(G) \leq|E| \text {. }
$$

Based on inequality (1), Ivančo and Jendroľ proposed a conjecture as follows [11]:

$$
\operatorname{tes}(G)=\max \left\{\left\lceil\frac{|E(G)|+2}{3}\right\rceil,\left\lceil\frac{\Delta(G)+1}{2}\right\rceil\right\} .
$$

Furthermore, some researchers have found exact values of tes of some graph classes. Readers could refer to [7], [11], [12], [14], [15] [10], [9], [18], [1], [8], [2], [13], and [16].

Many graph classes have used to model real problems. Such as in chemical structures, the graph classes that commonly used are cactus graphs, where atoms represent vertices and chemical bonds represent edges of the graphs ([6], [3], [17]). A cactus graph $G$ is a connected graph in which each edge lies on exactly one cycle. In other words, the cactus graph comprises some blocks where each block is either an edge or a cycle. The cactus graph is named $n$-uniform if each block is a cycle with the same order $n$. If each cycle of cactus graph $G$ has at most two cut-vertices and each cut-vertex is shared by exactly two cycles, then $G$ is called $n$-uniform cactus chain graph. The number of cycles of the cactus chain graph indicates the length of the chain graph.

Further, a tadpole graph $T(m, n)$ is graph which consists of a cycle graph $C_{m}$ and a path graph $P_{n}$ which is connected with a bridge. Whereas, a tadpole chain graph is a chain graph which have tadpole graphs in all blocks. In this paper, we investigate tes of some $n$-uniform cactus chain graphs $C\left(C_{n}^{r}\right)$ of length $r$ for some $n \equiv 0 \bmod 3$ and tes of tadpole chain graphs $T_{r}(4, n)$ and $T_{r}(5, n)$.

\section{Main Results}

The formula for tes of $n$-uniform cactus chain graph $C\left(C_{n}^{r}\right)$ is provided in Subsection 2.1. Meanwhile, the tes of tadpole chain graphs $T_{r}(4, n)$ and $T_{r}(5, n)$ are presented in Subsection 2.2.

\section{1. n-Uniform Cactus Chain Graphs}

The concept of $n$-uniform cactus chain graphs is given in Definition 2.1.

Definition 2.1. An n-uniform cactus graph $G$ is a cactus graph consisting of some blocks where each block is cycle $C_{n}$ of same size $n$. If each cycle of the cactus graph contains no more than two cut-vertices and every two blocks has exactly one common cut-vertex, then $G$ is called n-uniform cactus chain graph. The length of the cactus chain is indicated by the number of cycles in the chain. The n-uniform cactus chain graphs with length $r$ for any natural number $n$, denoted by $C\left(C_{n}^{r}\right)$, 
consists of blocks $B_{1}, B_{2}, \ldots, B_{r}$ where vertices on block $B_{i}$ are $\left\{a_{i}, u_{i}^{1}, u_{i}^{2} \ldots, u_{i}^{\left\lfloor\frac{n-2}{2}\right\rfloor}, v_{i}^{1}, v_{i}^{2}, \ldots\right.$, $\left.v_{i}^{\left\lceil\frac{n-2}{2}\right\rceil}\right\}$ for $i=1,2, \ldots, r$. Vertex $a_{i+1}$ is a cut vertex between two blocks $B_{i}$ and $B_{i+1}$. It means that $a_{i+1}$ lies on two blocks $B_{i}$ and $B_{i+1}$. Meanwhile, the edges of each block $B_{i}$ are $\left\{a_{i} u_{i}^{1}, u_{i}^{1} u_{i}^{2}, u_{i}^{2} u_{i}^{3}, \ldots, u_{i}^{\left\lfloor\frac{n-2}{2}\right\rfloor} a_{i+1}, a_{i} v_{i}^{1}, v_{i}^{1} v_{i}^{2}, \ldots, v_{i}^{\left\lceil\frac{n-2}{2}\right\rceil} a_{i+1}\right\}$.

Further, tes of some $n$-uniform cactus chain graphs $C\left(C_{n}^{r}\right)$ for $n \equiv 0 \bmod 3$ and length $r=$ 1, 2 is given in Lemma 2.1.

Lemma 2.1. Let $C\left(C_{n}^{r}\right)$ be n-uniform cactus chain graphs of length $r=1,2$ with $n \equiv 0 \bmod 3$ and $n \geq 6$. Then,

$$
\operatorname{tes}\left(C\left(C_{n}^{r}\right)\right)=\left\lceil\frac{n r+2}{3}\right\rceil .
$$

Proof. Let $n \equiv 0 \bmod 3$ and $n \geq 6$. The $n$-uniform cactus chain graphs $C\left(C_{n}^{r}\right)$ with length $r=$ 1,2 consist of two blocks $B_{1}$ and $B_{2}$ whose vertices and edges are $\left\{a_{i}, u_{i}^{1}, u_{i}^{2} \ldots, u_{i}^{\left\lfloor\frac{n-2}{2}\right\rfloor}, v_{i}^{1}, v_{i}^{2}, \ldots\right.$, $\left.v_{i}^{\left\lceil\frac{n-2}{2}\right\rceil}\right\}$ and $\left\{a_{i} u_{i}^{1}, u_{i}^{1} u_{i}^{2}, u_{i}^{2} u_{i}^{3}, \ldots, u_{i}^{\left\lfloor\frac{n-2}{2}\right\rfloor} a_{i+1}, a_{i} v_{i}^{1}, v_{i}^{1} v_{i}^{2}, \ldots, v_{i}^{\left\lceil\frac{n-2}{2}\right\rceil} a_{i+1}\right\}$, respectively, for $i=$ 1,2. The cut vertex $a_{2}$ is located two blocks $B_{1}$ and $B_{2}$

Based on (1), the bound of tes $\left(C\left(C_{n}^{r}\right)\right)$ is as follows:

$$
\left\lceil\frac{n r+2}{3}\right\rceil \leq \operatorname{tes}\left(C\left(C_{n}^{r}\right)\right) \leq n r .
$$

To prove tes $\left(C\left(C_{n}^{r}\right)\right) \leq\left\lceil\frac{n r+2}{3}\right\rceil$, we construct a total $k$-labeling $f: V \cup E \rightarrow\{1,2, \ldots, k\}$ with $k=\left\lceil\frac{n r+2}{3}\right\rceil$ and show that $f$ satisfies the required properties of an edge irregular total $k$-labeling. Let us consider two cases.

Case 1. For $n \equiv 3 \bmod 6$ and $n \geq 9$.

Labels of vertices are constructed as follows:

$$
\begin{aligned}
& f\left(a_{i}\right)=\frac{n}{3}(i-1)+1, i=1,2 . \\
& f\left(u_{i}^{j}\right)= \begin{cases}\frac{n}{3}(i-1)+j, & 1 \leq j \leq\left\lfloor\frac{n-2}{2}\right\rfloor, \quad n=9,15, i=1,2 \\
\frac{n}{3}(i-1)+j, & 1 \leq j \leq\left(\frac{n}{3}+1\right), \quad n \geq 21, i=1,2 .\end{cases}
\end{aligned}
$$

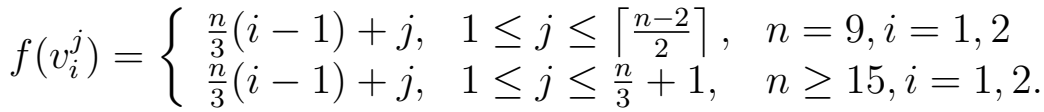

$$
\begin{aligned}
& f\left(u_{i}^{\frac{n}{3}+2}\right)=f\left(u_{i}^{\frac{n}{3}+3}\right)=\ldots=f\left(u_{i}^{\left\lfloor\frac{n-2}{2}\right\rfloor}\right)=\left\lceil\frac{n i+2}{3}\right\rceil, i=1,2 \text {, for } n \geq 21 \text {; } \\
& f\left(v_{i}^{\frac{n}{3}+2}\right)=f\left(v_{i}^{\frac{n}{3}+3}\right)=\ldots=f\left(v_{i}^{\left\lceil\frac{n-2}{2}\right\rceil}\right)=\left\lceil\frac{n i+2}{3}\right\rceil, i=1,2 \text {, for } n \geq 15 \text {. }
\end{aligned}
$$

Furthermore, we define labels of edges as follows: 


$$
\begin{aligned}
& f\left(a_{i} u_{i}^{1}\right) \quad=\frac{n}{3}(i-1)+1, i=1,2, \\
& f\left(a_{i} v_{i}^{1}\right) \quad=\frac{n}{3}(i-1)+3, i=1,2 . \\
& f\left(a_{i+1} u_{i}^{\left\lfloor\frac{n-2}{2}\right\rfloor}\right)=\frac{n}{3}(i)-1, i=1,2 \\
& f\left(a_{i+1} v_{i}^{\left\lceil\frac{n-2}{2}\right\rceil}\right)=\frac{n}{3}(i), i=1,2 \text {, } \\
& f\left(u_{i}^{1} u_{i}^{2}\right) \quad=\frac{n}{3}(i-1)+1, i=1,2 . \\
& f\left(u_{i}^{j} u_{i}^{j+1}\right)= \begin{cases}\frac{n}{3}(i-1)+2, \quad i=1,2 ; 2 \leq j \leq \frac{n}{3}-1, & n=9, \\
\frac{n}{3}(i-1)+2, \quad i=1,2 ; 2 \leq j \leq \frac{n}{3}, & n \geq 15 .\end{cases} \\
& f\left(u_{i}^{\frac{n}{3}+j} u_{i}^{\frac{n}{3}+j+1}\right)=\frac{n}{3}(i-1)+2(j+1), i=1,2,1 \leq j \leq\left\lfloor\frac{n-2}{2}\right\rfloor-\left(\frac{n}{3}+1\right), n \geq 21 . \\
& f\left(v_{i}^{j} v_{i}^{j+1}\right)= \begin{cases}\frac{n}{3}(i-1)+3, \quad i=1,2,1 \leq j \leq \frac{n}{3}, & n=9 \\
\frac{n}{3}(i-1)+3, & i=1,2,1 \leq j \leq \frac{n}{3}+1, \quad n \geq 15\end{cases} \\
& f\left(v_{i}^{\frac{n}{3}+j} v_{i}^{\frac{n}{3}+j+1}\right)=\frac{n}{3}(i-1)+2 j+1, i=1,2 \text {, with } 2 \leq j \leq\left\lfloor\frac{n-2}{2}\right\rfloor-\left(\frac{n}{3}\right) \text { and } n \geq 21 \text {; }
\end{aligned}
$$

By using above labeling, we get the edge weights as follows:

$$
\begin{aligned}
& w t\left(a_{i} u_{i}^{1}\right)=n(i-1)+3, i=1,2 ; \\
& w t\left(a_{i} v_{i}^{1}\right)=n(i-1)+5, i=1,2 ; \\
& w t\left(a_{i+1} u_{i}^{\left\lfloor\frac{n-2}{2}\right\rfloor}\right)=n i ; i=1,2, n=9 ; \\
& w t\left(a_{i+1} u_{i}^{\left\lfloor\frac{n-2}{2}\right\rfloor}\right)=n i+1 ; i=1,2, n \geq 15 ; \\
& w t\left(a_{i+1} v_{i}^{\left\lceil\frac{n-2}{2}\right\rceil}\right)=n i+2 ; i=1,2, n \geq 9 ; \\
& w t\left(u_{i}^{1} u_{i}^{2}\right)=n(i-1)+4, i=1,2 ; n \geq 9 ; \\
& w t\left(u_{i}^{j} u_{i}^{j+1}\right)=n(i-1)+2 j+3, i=1,2, \text { with } 2 \leq j \leq \frac{n}{3}-1 \text { and } n=9 ; \\
& w t\left(u_{i}^{j} u_{i}^{j+1}\right)=n(i-1)+2 j+3, i=1,2, \text { with } 2 \leq j \leq \frac{n}{3} \text { and } n \geq 15 ; \\
& w t\left(u_{i}^{\frac{n}{3}+j} u_{i}^{\frac{n}{3}+j+1}\right)=2\left\lceil\frac{n i+2}{3}\right\rceil+\frac{n}{3}(i-1)+2(j+1), i=1,2, \\
& \text { with } 1 \leq j \leq\left\lfloor\frac{n-2}{2}\right\rfloor-\left(\frac{n}{3}+1\right) \text { and } n \geq 21 . \\
& w t\left(v_{i}^{j} v_{i}^{j+1}\right)=n(i-1)+2 j+4, i=1,2, \text { with } 1 \leq j \leq \frac{n}{3} \text { and } n \geq 9 ; \\
& w t\left(v_{i}^{\frac{n}{3}+j} v_{i}^{\frac{n}{3}+j+1}\right)=2\left\lceil\frac{n i+2}{3}\right\rceil+\frac{n}{3}(i-1)+2 j+1, i=1,2, \\
& \text { with } 1 \leq j \leq\left\lfloor\frac{n-2}{2}\right\rfloor-\left(\frac{n}{3}\right) \text { and } n \geq 15 .
\end{aligned}
$$

We verify that all labels of vertices and edges are less then or equal to $\left\lceil\frac{n r+2}{3}\right\rceil$ and the weights of edges are all distinct. Thus, tes $\left(C\left(C_{n}^{r}\right)\right)=\left\lceil\frac{n r+2}{3}\right\rceil$ for $r=1,2, n$ is odd, $n \geq 9$ and $n \equiv 0 \bmod 3$. 
Case 2. For $n \equiv 0 \bmod 6, n \geq 6$, and $r=1,2$.

The vertex labels are described as follows:

$$
\begin{aligned}
& f\left(a_{i}\right)=\frac{n}{3}(i-1)+1, i=1,2, n \geq 6 .
\end{aligned}
$$

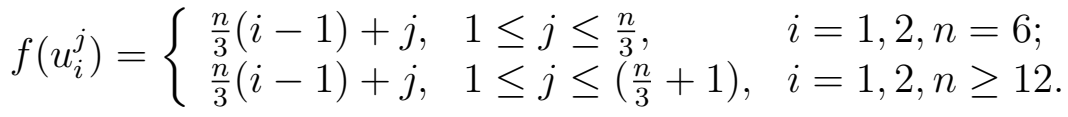

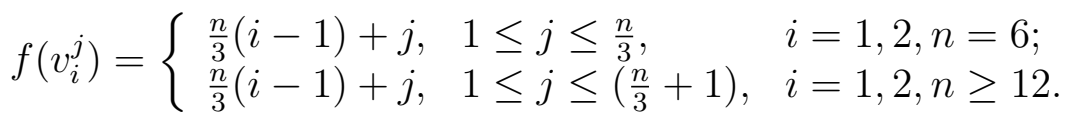

$$
\begin{aligned}
& f\left(u_{i}^{\frac{n}{3}+2}\right)=f\left(u_{i}^{\frac{n}{3}+3}\right)=\ldots=f\left(u_{i}^{\left(\frac{n-2}{2}\right)}\right)=\left\lceil\frac{n i+2}{3}\right\rceil, i=1,2 \text {, for } n \geq 18 \text {; } \\
& f\left(v_{i}^{\frac{n}{3}+2}\right)=f\left(v_{i}^{\frac{n}{3}+3}\right)=\ldots=f\left(v_{i}^{\left(\frac{n-2}{2}\right)}\right)=\left\lceil\frac{n i+2}{3}\right\rceil, i=1,2 \text {, for } n \geq 18 \text {. }
\end{aligned}
$$

The edge labels are given as follows:

$$
\begin{aligned}
& f\left(a_{i}^{1} u_{i}^{1}=\frac{n}{3}(i-1)+1, i=1,2\right. \\
& f\left(a_{i}^{1} v_{i}^{1}\right)=\frac{n}{3}(i-1)+2, i=1,2 \text {; } \\
& f\left(a_{i+1} u_{i}^{\left\lfloor\frac{n-2}{2}\right\rfloor}\right)= \begin{cases}\frac{n}{3}(i)-1, & i=1,2, \quad n \geq 12 \\
\frac{n}{3}(i), & i=1,2, \quad n=6\end{cases} \\
& f\left(a_{i+1} v_{i}^{\left\lceil\frac{n-2}{2}\right\rceil}\right)= \begin{cases}\frac{n}{3}(i), & i=1,2, \quad n \geq 12 \\
\frac{n}{3}(i)+1, & n=6 .\end{cases} \\
& f\left(u_{i}^{j} u_{i}^{j+1}\right)=\left\{\begin{array}{lll}
\frac{n}{3}(i-1)+2, & 1 \leq j \leq \frac{n}{3}, i=1,2, & n \geq 12 \\
& 1 \leq j \leq \frac{n}{3}-1, & n=6 .
\end{array}\right. \\
& f\left(v_{i}^{j} v_{i}^{j+1}\right)=\left\{\begin{array}{lll}
\frac{n}{3}(i-1)+3, & 1 \leq j \leq \frac{n}{3}, i=1,2, & n \geq 12 \\
& 1 \leq j \leq \frac{n}{3}-1, & n=6
\end{array}\right. \\
& f\left(u_{i}^{\frac{n}{3}+j} u_{i}^{\frac{n}{3}+j+1}\right)=\frac{n}{3}(i-1)+2 j+1, i=1,2,1 \leq j \leq\left\lceil\frac{n-2}{2}\right\rceil-\left(\frac{n}{3}+1\right) \text { for } n \geq 18 . \\
& f\left(v_{i}^{\frac{n}{3}+j} v_{i}^{\frac{n}{3}+j+1}\right) \quad=\frac{n}{3}(i-1)+2(j+1), i=1,2,1 \leq j \leq\left\lceil\frac{n-2}{2}\right\rceil-\left(\frac{n}{3}+1\right) \text { for } n \geq 18 \text {; }
\end{aligned}
$$

By using the above labeling, we get the weight of edges as follows:

$$
\begin{aligned}
& w t\left(a_{i} u_{i}^{1}\right)=n(i-1)+3, i=1,2 ; n \geq 6 . \\
& w t\left(a_{i} v_{i}^{1}\right)=n(i-1)+4, i=1,2 ; n \geq 6 . \\
& w t\left(a_{i+1} u_{i}^{\left(\frac{n-2}{2}\right)}\right)=n i+1 ; i=1,2, n \geq 6 ; \\
& w t\left(a_{i+1} v_{i}^{\left(\frac{n-2}{2}\right)}\right)=n i+2, i=1,2, n \geq 6 ; \\
& \left.w t\left(u_{i}^{j} u_{i}^{j+1}\right)=n(i-1)+2 j+3, i=1,2,1 \leq j \leq \frac{n}{3} \text { (for } n \geq 12\right), \\
& \text { and } 1 \leq j \leq \frac{n}{3}-1(\text { for } n=6) ; \\
& w t\left(u_{i}^{\frac{n}{3}+j} u_{i}^{\frac{n}{3}+j+1}\right)=2\left\lceil\frac{n i+2}{3}\right\rceil+\frac{n}{3}(i-1)+2 j+1, i=1,2, \\
& \text { with } 1 \leq j \leq\left\lceil\frac{n-2}{2}\right\rceil-\left(\frac{n}{3}+1\right), n \geq 18 .
\end{aligned}
$$




$$
\begin{aligned}
& w t\left(v_{i}^{j} v_{i}^{j+1}\right)=n(i-1)+2 j+4, i=1,2, \text { with } 1 \leq j \leq \frac{n}{3} \text { for } n \geq 12 ; \\
& \text { and } 1 \leq j \leq \frac{n}{3}-1 \text { for } n=6 ; \\
& w t\left(v_{i}^{\frac{n}{3}+j} v_{i}^{\frac{n}{3}+j+1}\right)=2\left\lceil\frac{n i+2}{3}\right\rceil+\frac{n}{3}(i-1)+2(j+1), i=1,2, \\
& \text { with } 1 \leq j \leq\left\lceil\frac{n-2}{2}\right\rceil-\left(\frac{n}{3}+1\right) \text { and } n \geq 18 .
\end{aligned}
$$

So the vertex and edge labels are at most $\left\lceil\frac{n r+2}{3}\right\rceil$ and each edge has a distinct weight. Thus, tes $\left(C\left(C_{n}^{r}\right)\right)=\left\lceil\frac{n r+2}{3}\right\rceil$ for $n \equiv 0 \bmod 6, n \geq 6$, and $r=1,2$.

One of the objectives of this paper is to prove tes of some $n$-uniform cactus chain graphs $\left(C\left(C_{n}^{r}\right)\right)$ for $n \equiv 0 \bmod 3$ as presented in Theorem 2.1.

Theorem 2.1. Let $\left(C\left(C_{n}^{r}\right)\right)$ be $n$-uniform cactus chain graphs with $n \equiv 0 \bmod 3, n \geq 6$ and length $r \geq 1$. Then,

$$
\operatorname{tes}\left(C\left(C_{n}^{r}\right)\right)=\left\lceil\frac{n r+2}{3}\right\rceil
$$

Proof. We prove statement (2) by induction on $n$ and $r$.

1. Statement (2) is proved by induction on $n$.

According to Lemma 2.1, tes $\left(C\left(C_{6}^{r}\right)\right)=\left\lceil\frac{(6 r+2)}{3}\right\rceil$. Therefore, statement (2) is true for $n=6$.

Based on Lemma 2.1, we also get tes $\left(C\left(C_{n}^{1}\right)\right)=\left\lceil\frac{(n+2)}{3}\right\rceil$. Hence, the theorem holds for base case $r=1, n=6$. Assume that Statement (2) is true for some $k$, i.e.,

$$
\operatorname{tes}\left(C\left(C_{k}^{r}\right)\right)=\left\lceil\frac{k r+2}{3}\right\rceil .
$$

We will check the the statement for $n=k+3$ by considering two cases.

(a) Case 1: for $r=1$.

By means of Lemma 2.1, we get tes $\left(C\left(C_{k+3}^{1}\right)\right)=\left\lceil\frac{(k+3)+2}{3}\right\rceil$. Thus, the statement is true for $r=1$.

(b) Case 2: for $r>1$.

Let cycle $C_{k}$ on the each block is $v_{1} e_{1} v_{2} e_{2} v_{3} \ldots v_{k-1} e_{k-1} v_{k} e_{k} v_{1}$. We can get cycle $C_{k+3}$ on each block by spliting edge $e_{k-1}$ into three edges with three new vertices $x_{1}, x_{2}, x_{3}$ [4]. Based on assumption (3), there is an edge irregular total $k^{\prime}$-labeling $f$ on $r$-blocks with $k^{\prime}=\left\lceil\frac{k r+2}{3}\right\rceil$. Define labels for $v_{k-1}$ and $v_{k}$ on $r$-blocks as follows: $f\left(v_{k-1}^{r}\right)=k^{\prime}-1=\left\lceil\frac{k r+2}{3}\right\rceil-1, f\left(v_{k}^{r}\right)=k^{\prime}=\left\lceil\frac{k r+2}{3}\right\rceil$ with $r>1$. We construct optimal labeling in $C_{k+3}$ on $r$-blocks by using procedures as follows:

i. define labels of vertices $f\left(x_{1}^{r}\right)=k^{\prime}+r, f\left(x_{2}^{r}\right)=k^{\prime}+r, f\left(x_{3}^{r}\right)=k^{\prime}+r$;

ii. define labels of edges $f\left(v_{k-1}^{r} x_{1}^{r}\right)=k^{\prime}+r, f\left(x_{1}^{r} x_{2}^{r}\right)=k^{\prime}+r, f\left(x_{2}^{r} x_{3}^{r}\right)=k^{\prime}$, $f\left(x_{3}^{r} v_{k}^{r}\right)=k^{\prime}$. 
By means of total labeling $f$ and the above procedures, we get different weights of edges on $C\left(C_{k+3}^{r}\right)$. Furthermore, the maximum label of vertices and edges on $C\left(C_{k+3}^{r}\right)$ is $k^{\prime}+r=\left\lceil\frac{k r+2}{3}\right\rceil+\frac{3 r}{3}=\left\lceil\frac{(k+3) r+2}{3}\right\rceil$. Thus, statement (2) is true for $n$.

2. Statement (2) is proved by induction on $r$.

Based on Lemma 2.1, tes $\left(C\left(C_{n}^{1}\right)\right)=\left\lceil\frac{(n+2)}{3}\right\rceil$. Hence, the theorem is true for $r=1$. Assume that the theorem is true for $r$, i.e., tes $\left(C\left(C_{n}^{r}\right)\right)=\left\lceil\frac{n r+2}{3}\right\rceil$. We need to verify the theorem for $r+1$. The cactus chain graph $C\left(C_{n}^{r+1}\right)$ consists of $(r+1)$ blocks, i.e., $B_{1}, B_{2}, \ldots, B_{r}, B_{r+1}$. By using the assumption, there exists an edge irregular total $k$-labeling $f$ on $r$ blocks with $k=\left\lceil\frac{n r+2}{3}\right\rceil$. We construct procedures to label vertices and edges on block $B_{r+1}$ through total labeling $f$ as follows:

(a) labels of vertices $u_{r+1}^{1}, u_{r+1}^{2} \ldots, u_{r+1}^{\left\lfloor\frac{n-2}{2}\right\rfloor}, v_{r+1}^{1}, v_{r+1}^{2}, \ldots, v_{r+1}^{\left\lceil\frac{n-2}{2}\right\rceil}, a_{r+2}$ on block $B_{r+1}$ are acquired by adding a constant number $\frac{n}{3}$ to each label $f\left(u_{r}^{1}\right), f\left(u_{r}^{2}\right) \ldots, f\left(u_{r}^{\left\lfloor\frac{n-2}{2}\right\rfloor}\right)$, $f\left(v_{r}^{1}\right), f\left(v_{r}^{2}\right), \ldots, f\left(v_{r}^{\left\lceil\frac{n-2}{2}\right\rceil}\right), f\left(a_{r+1}\right)$ on block $B_{r}$;

(b) labels of edges $a_{r+1} u_{r+1}^{1}, u_{r+1}^{1} u_{r+1}^{2}, u_{r+1}^{2} u_{r+1}^{3}, \ldots, u_{r+1}^{\left\lfloor\frac{n-2}{2}\right\rfloor} a_{r+2}, a_{r+1} v_{r+1}^{1}, v_{r+1}^{1} v_{r+1}^{2}$, $\ldots, v_{r+1}^{\left\lceil\frac{n-2}{2}\right\rceil} a_{r+2}$ on block $B_{r+1}$ are derived by adding a constant number $\frac{n}{3}$ to each label of edge $a_{r} u_{r}^{1}, u_{r}^{1} u_{r}^{2}, u_{r}^{2} u_{r}^{3}, \ldots, u_{r}^{\left\lfloor\frac{n-2}{2}\right\rfloor} a_{r+1}, a_{r} v_{r}^{1}, v_{r}^{1} v_{r}^{2}, \ldots, v_{r}^{\left\lceil\frac{n-2}{2}\right\rceil} a_{r+1}$ on block $B_{r}$.

All weights of edges on block $B_{r+1}$ are obtained by adding a constant number $n$ to each weight of edge on block $B_{r}$. Since we have different weights of edges on block $B_{r}$ under labeling $f$, we also obtain different weights of edges on block $B_{r+1}$. Moreover, labels of vertices and edges of $C\left(C_{n}^{r}\right)$ are not more than $\left\lceil\frac{n r+2}{3}\right\rceil$. Hence, the largest label on block $B_{r+1}$ is $\left\lceil\frac{n r+2}{3}\right\rceil+\left\lceil\frac{n}{3}\right\rceil=\left\lceil\frac{n(r+1)+2}{3}\right\rceil$ which shows the theorem holds for $r+1$. Thus, tes $\left(C\left(C_{n}^{r}\right)\right)=\left\lceil\frac{n r+2}{3}\right\rceil$ is true for all $r$ and $n \equiv 0 \bmod 3$.

In Figure 1, we give an example of edge irregular total 21-labeling of $C\left(C_{15}^{4}\right)$ for which $\operatorname{tes}\left(C\left(C_{15}^{4}\right)\right)=21$.

\subsection{Tadpole Chain Graphs}

In this section, we provide formulas for tes of some tadpole chain graphs, i.e. tes of $T_{r}(4, n)$ and $T_{r}(5, n)$. Firstly, we give definition of the tadpole chain graphs.

Definition 2.2. A tadpole graph $T(m, n)$ is graph which consists of a cycle graph of $m$ vertices connected with a bridge to a path graph of $n$ vertices. Further, a tadpole chain graph of length $r$, symbolized by $T_{r}(m, n)$, is an m-uniform cactus chain graph where cycle $C_{m}$ on each block is connected with a bridge to a path graph $P_{n}$ of $n$ vertices where the length $r$ indicates the number of tadpole graphs on the chain. 


\section{Computing total edge irregularity strength of ... $\quad$ | I. Rosyida and D. Indriati}

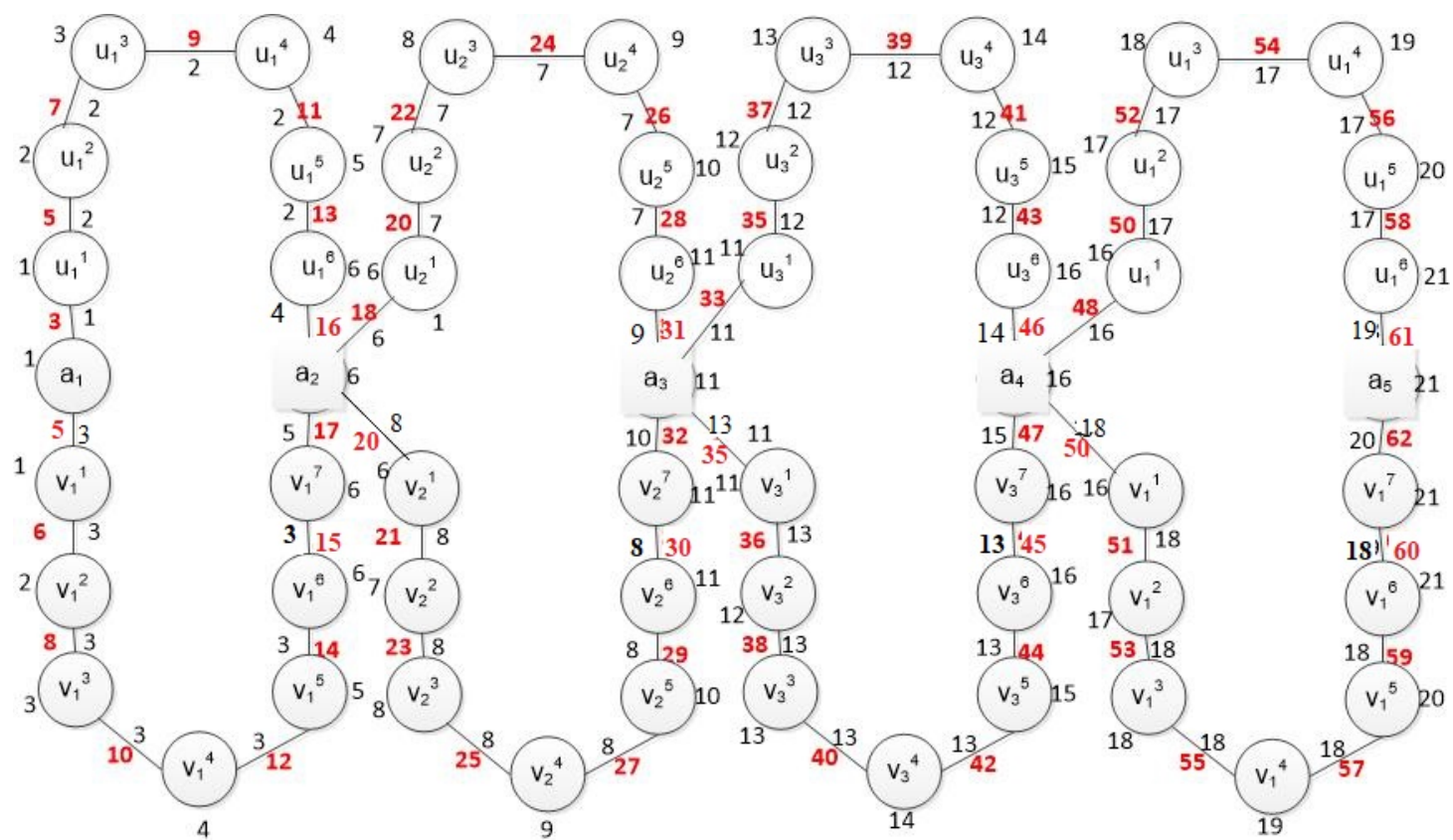

Figure 1. Edge irregular total 21-labeling of $C\left(C_{15}^{4}\right)$

Based on Definition 2.2, tadpole chain graph $\left(T_{r}(4, n)\right)$ consists of $r$ blocks $B_{1}, B_{2}, \ldots, B_{r}$ where each block is tadpole graph $T(4, n)$. Also, tadpole chain graph $\left(T_{r}(5, n)\right)$ consists of $r$ blocks $B_{1}, B_{2}, \ldots, B_{r}$ where each block is tadpole graph $T(5, n)$.

Lemma 2.2. Given a tadpole chain graph $\left(T_{r}(4, n)\right)$ of length $r$ with $r=1,2,3, n \equiv 2 \bmod 3$, and $n \geq 5$. Then,

$$
\operatorname{tes}\left(T_{r}(4, n)\right)=\left\lceil\frac{(4+n) r+2}{3}\right\rceil .
$$

Proof. Tadpole chain graph $T_{r}(4, n)$ consists $4+n$ vertices and $4+n$ edges on each block. Let $u_{i}, v_{i}, v_{i+1}, x_{i}$ be vertices located on cycle $C_{4}$ of each block $B_{i}$ where $v_{i+1}$ is a cut vertex between $B_{i}$ and $B_{i+1}$. Let $y_{i}^{1}, y_{i}^{2}, \ldots, y_{i}^{n}$ be vertices of the path connected with a bridge to vertex $x_{i}$. The edges of $T_{r}(4, n)$ on block $B_{i}$ are $\left\{u_{i}, v_{i}, u_{i} v_{i+1}, v_{i} x_{i}, v_{i+1} x_{i}, x_{i} y_{i}^{1}\right\} \cup\left\{y_{i}^{j} y_{i}^{j+1}, j=1,2, \ldots, n-1\right\}$.

Based on (1), the lower bound for tes $\left(T_{r}(4, n)\right)$ is as follows:

$$
\left\lceil\frac{(4+n) r+2}{3}\right\rceil \leq \operatorname{tes}\left(T_{r}(4, n)\right) \leq(4+n) r .
$$

We will verify an existence of a total edge irregular $k$-labeling $f: V \cup E \rightarrow\{1,2, \ldots, k\}$ where $k$ is $\left\lceil\frac{(4+n) r+2}{3}\right\rceil$ to get upper bound tes $\left(T_{r}(4, n)\right) \leq\left\lceil\frac{(4+n) r+2}{3}\right\rceil$. Let us consider two cases.

Case 1. For $n \equiv 5 \bmod 6, n \geq 5$, and $r=1,2,3$.

We construct labels of vertices in the following way: 


$$
\begin{aligned}
& f\left(u_{1}\right)=1, f\left(v_{1}\right)=1, f\left(v_{2}\right)=3 ; f\left(x_{1}\right)=2 . \\
& f\left(v_{3}\right)=f\left(v_{2}\right)+\left(\frac{n+4}{3}\right), n \geq 5 \text {, } \\
& f\left(v_{4}\right)=f\left(v_{3}\right)+\left(\frac{n+4}{3}\right), n \geq 5 \text {. } \\
& f\left(u_{2}\right)=3+\frac{(n+1)}{2} ; f\left(u_{3}\right)=4+n, n \geq 5 \text {. } \\
& f\left(x_{2}\right)=3+\left\lceil\frac{(n+2)}{2}\right\rceil ; f\left(x_{3}\right)=5+n, n \geq 5 \text {. } \\
& \begin{array}{l}
f\left(y_{1}^{j}\right)=\left\{\begin{array}{lll}
2, & 1 \leq j \leq\left(\frac{n+4}{3}\right)-1, & n \geq 5 ; \\
3, & j=\frac{n+4}{3}, \frac{n+4}{3}+1, & n \geq 5 ; \\
{\left[\frac{(4+n)+2}{3}\right\rceil,} & j=n, & n \geq 5 .
\end{array}\right. \\
f\left(y_{2}^{j}\right)=\left\{\begin{array}{lll}
{\left[\frac{(4+n) \cdot 2+2}{3}\right],} & 1 \leq j \leq n, \quad n=5,11 ; \\
\left.\frac{(4+n) \cdot 2+2}{3}\right]-\left\lfloor\left(\frac{n-8}{6}\right)\right\rfloor, & j=1,2, & n \geq 17 ; \\
\left.\frac{(4+n) \cdot 2+2}{3}\right\rceil, & \frac{n-8}{3} \leq j \leq n, & n \geq 17 .
\end{array}\right.
\end{array} \\
& f\left(y_{1}^{\frac{n+4}{3}+j}\right)=3+\left\lfloor\frac{j}{2}\right\rfloor, 2 \leq j \leq n-\left(\frac{n+10}{3}\right)+1, n \geq 11 \\
& f\left(y_{2}^{2+j}\right)=\left[\frac{(4+n) \cdot 2+2}{3}\right]-\left\lfloor\left(\frac{n-8}{6}\right)\right\rfloor+\left\lfloor\frac{j+1}{2}\right\rfloor, 1 \leq j \leq \frac{(n-11)}{3}, n \geq 23 \text {; } \\
& f\left(y_{3}^{j}\right) \quad=\left\lceil\frac{(4+n) \cdot 3+2}{3}\right\rceil, 1 \leq j \leq n, n \geq 5 \text {. }
\end{aligned}
$$

Further, we provide labels of edges as follows:

$$
\begin{aligned}
& f\left(u_{1} v_{1}\right)=1, f\left(x_{1} v_{1}\right)=1, f\left(u_{1} v_{2}\right)=1, f\left(x_{1} v_{2}\right)=1 ; \\
& f\left(u_{2} v_{2}\right)=\left[\frac{(4+n) \cdot 2+2}{3}\right]-4, f\left(x_{2} v_{2}\right)=\left[\frac{(4+n) \cdot 2+2}{3}\right]-4, n=5 ; \\
& f\left(u_{2} v_{3}\right)=\left[\frac{(4+n) \cdot 2+2}{3}\right]-5, f\left(x_{2} v_{3}\right)=\left[\frac{(4+n) \cdot 2+2}{3}\right]-5, n=5 ; \\
& \left.f\left(u_{2} v_{2}\right)=-\frac{(4+n) \cdot 2+2}{3}\right]-\left(\frac{n+19}{6}\right), n \geq 11 ; \\
& \left.f\left(x_{2} v_{2}\right)=\frac{(4+n) \cdot 2+2}{3}\right]-\left(\frac{n+19}{6}\right), n \geq 11 ; \\
& \left.f\left(u_{2} v_{3}\right)=\frac{(4+n) \cdot 2+2}{3}\right]-\left(\frac{n+5}{2}\right), n \geq 11 ; \\
& \left.f\left(x_{2} v_{3}\right)=-\frac{(4+n) \cdot 2+2}{3}\right]-\left(\frac{n+5}{2}\right), n \geq 11 ; \\
& \left.f\left(u_{3} v_{3}\right)=\frac{(4+n) \cdot 3+2}{3}\right]-\left(\frac{n+7}{3}\right), n \geq 5 ; \\
& \left.f\left(x_{3} v_{3}\right)=\frac{(4+n) \cdot 3+2}{3}\right\rceil-\left(\frac{n+7}{3}\right), n \geq 5 ; \\
& f\left(u_{3} v_{4}\right)=\left(\frac{n+10}{3}\right) ; f\left(x_{3} v_{4}\right)=\left(\frac{n+10}{3}\right) ; n \geq 5 . \\
& f\left(x_{1} y_{1}^{1}\right)=3, n \geq 5 ; \\
& f\left(y_{1}^{j} y_{1}^{j+1}\right)=\left\lceil\frac{(4+n)+2}{3}\right\rceil, 1 \leq j \leq n, n=5 ; \\
& f\left(x_{2} y_{2}^{1}\right)=2, n=5 ; \\
& f\left(y_{2}^{j} y_{2}^{j+1}\right)=j+2,1 \leq j \leq n-1, n=5 . \\
& f\left(y_{1}^{j} y_{1}^{j+1}\right)=3+j, 1 \leq j \leq\left(\frac{n-5}{3}\right), n \geq 11 ; \\
& f\left(y_{1}^{j} y_{1}^{j+1}\right)=\left\lceil\frac{(4+n)+2}{3}\right\rceil,\left(\frac{n-5}{3}+1\right) \leq j \leq n-1, n \geq 11 ;
\end{aligned}
$$




$$
\begin{aligned}
& f\left(x_{2} y_{2}^{1}\right)=1, n \geq 11 ; \\
& f\left(y_{2}^{j} y_{2}^{j+1}\right)=1,1 \leq j \leq\left(\frac{n-5}{3}\right)-1, n \geq 11 \\
& f\left(y_{2}^{j} y_{2}^{j+1}\right)=j+2-\left(\frac{n-5}{3}\right), \frac{n-5}{3} \leq j \leq n-1 . \\
& f\left(x_{3} y_{3}^{1}\right)=5, n \geq 5 ; \\
& f\left(y_{3}^{j} y_{3}^{j+1}\right)=j+5,1 \leq j \leq n-1, n \geq 5 .
\end{aligned}
$$

Case 2. For $n \equiv 2 \bmod 6, n \geq 8$, and $r=1,2,3$.

We construct labels of vertices and edges as follows:

$$
\begin{aligned}
& f\left(u_{1}\right)=1, f\left(v_{1}\right)=1, f\left(v_{2}\right)=3 ; f\left(x_{1}\right)=2 ; n \geq 8 \text {; } \\
& f\left(u_{2}\right)=3+\frac{n+2}{2}, n \geq 8 \\
& f\left(x_{2}\right)=3+\frac{n+4}{2}, n \geq 8 \\
& f\left(u_{3}\right)=4+n ; f\left(x_{3}\right)=5+n, n \geq 8 \\
& f\left(v_{3}\right)=f\left(v_{2}\right)+\frac{n+4}{3} ; f\left(v_{4}\right)=f\left(\bar{v}_{3}\right)+\frac{n+4}{3}, n \geq 8 \text {. } \\
& \begin{array}{l}
f\left(y_{1}^{j}\right)=\left\{\begin{array}{lll}
2, & 1 \leq j \leq\left(\frac{n+4}{3}\right)-1, & n \geq 8, \\
3, & \frac{n+4}{3} \leq j \leq \frac{n+4}{3}+1, & n \geq 8, \\
{\left[\frac{(4+n)+2}{3}\right],} & j=n, & n \geq 8
\end{array}\right. \\
f\left(y_{2}^{j}\right)=\left\{\begin{array}{lll}
{\left[\frac{(4+n) \cdot 2+2}{3}\right],} & 1 \leq j \leq n, & n=8 ; \\
\left.\frac{(4+n) \cdot 2+2}{3}\right]-\left(\frac{n-8}{6}\right), & j=1, & n \geq 14 ; \\
\left.\frac{(4+n) \cdot 2+2}{3}\right\rceil, & \frac{n-8}{3} \leq j \leq n, & n \geq 14 .
\end{array}\right.
\end{array} \\
& f\left(y_{1}^{\frac{n+4}{3}+j}\right)=3+\left\lfloor\frac{j}{2}\right\rfloor, 2 \leq j \leq n-\left(\frac{n+10}{3}\right)+1, n \geq 8 \\
& f\left(y_{2}^{1+j}\right)=\left\lceil\frac{(4+n) \cdot 2+2}{3}\right\rceil-\left\lfloor\left(\frac{n-8}{6}\right)\right\rfloor+\left\lceil\frac{j}{2}\right\rceil, 1 \leq j \leq\left(\frac{n-8}{3}\right)-2, n \geq 20 \text {; } \\
& f\left(y_{3}^{j}\right)=\left\lceil\frac{(4+n) \cdot 3+2}{3}\right\rceil, 1 \leq j \leq n, n \geq 8 \text {. } \\
& f\left(u_{1} v_{1}\right)=1, f\left(x_{1} v_{1}\right)=1, f\left(u_{1} v_{2}\right)=1, f\left(x_{1} v_{2}\right)=1, n \geq 8 \text {; } \\
& f\left(u_{2} v_{2}\right)=\left[\frac{(4+n) \cdot 2+2}{3}\right]-5, n=8 \text {; } \\
& f\left(x_{2} v_{2}\right)=\left[\frac{(4+n) \cdot 2+2}{3}\right]-5, n=8 \text {; } \\
& f\left(u_{2} v_{3}\right)=\left[\frac{(4+n) \cdot 2+2}{3}\right]-7, n=8 \text {; } \\
& f\left(x_{2} v_{3}\right)=\left[\frac{(4+n) \cdot 2+2}{3}\right]-7, n=8 \text {; } \\
& f\left(u_{2} v_{2}\right)=\left[\frac{(4+n) \cdot 2+2}{3}\right]-\left(\frac{n+22}{6}\right), n \geq 14 \text {; } \\
& f\left(x_{2} v_{2}\right)=\left[\frac{(4+n) \cdot 2+2}{3}\right]-\left(\frac{n+22}{6}\right), n \geq 14 \text {; } \\
& f\left(u_{2} v_{3}\right)=\frac{(4+n) \cdot 2+2}{3}-\left(\frac{n+6}{2}\right), n \geq 14 \text {; } \\
& f\left(x_{2} v_{3}\right)=\left\lceil\frac{(4+n) \cdot 2+2}{3}\right\rceil-\left(\frac{n+6}{2}\right), n \geq 14 \text {; }
\end{aligned}
$$




$$
\begin{aligned}
& f\left(u_{3} v_{3}\right)=\left[\frac{(4+n) \cdot 3+2}{3}\right]-\left(\frac{n+7}{3}\right), n \geq 8 ; \\
& f\left(x_{3} v_{3}\right)=\left\lceil\frac{(4+n) \cdot 3+2}{3}\right\rceil-\left(\frac{n+7}{3}\right), n \geq 8 ; \\
& f\left(u_{3} v_{4}\right)=f\left(x_{3} v_{4}\right)=\frac{(n+10)}{3}, n \geq 8 ; \\
& f\left(x_{1} y_{1}^{1}\right)=3 ; n \geq 8 ; \\
& f\left(y_{1}^{1} y_{1}^{2}\right)=4 ; f\left(y_{1}^{j} y_{1}^{j+1}\right)=\left\lceil\frac{(4+n)+2}{3}\right\rceil, 2 \leq j \leq n-1, n=8 ; \\
& f\left(y_{1}^{j} y_{1}^{j+1}\right)=3+j, 1 \leq j \leq\left(\frac{n-8}{3}+1\right), n \geq 14 ; \\
& f\left(y_{1}^{j} y_{1}^{j+1}\right)=\left\lceil\frac{(4+n)+2}{3}\right\rceil,\left(\frac{n-8}{3}+2\right) \leq j \leq n-1, n \geq 14 ; \\
& f\left(x_{2} y_{2}^{1}\right)=1 ; n \geq 8 ; \\
& f\left(y_{2}^{j} y_{2}^{j+1}\right)=j+1,1 \leq j \leq n-1, n=8 ; \\
& f\left(y_{2}^{j} y_{2}^{j+1}\right)=1,1 \leq j \leq\left(\frac{n-8}{3}\right), n \geq 14 ; \\
& f\left(y_{2}^{j} y_{2}^{j+1}\right)=j+1-\left(\frac{n-8}{3}\right), \frac{n-8}{3}+1 \leq j \leq n-1, n \geq 14 ; \\
& f\left(x_{3} y_{1}^{1}\right)=5 ; f\left(y_{3}^{j} y_{3}^{j+1}\right)=5+j ; 1 \leq j \leq n-1, n \geq 8 ;
\end{aligned}
$$

In both two cases, we get the edge weights in the following:

$$
\begin{aligned}
& w t\left(u_{1} v_{1}\right)=3 ; w t\left(x_{1} v_{1}\right)=4 ; w t\left(u_{1} v_{2}\right)=5 ; n \geq 5 \\
& w t\left(x_{1} v_{2}\right)=6 ; w t\left(x_{1} y_{1}^{1}\right)=7 ; n \geq 5 ; \\
& w t\left(y_{1}^{j} y_{1}^{j+1}\right)=7+j, 1 \leq j \leq n-1 ; n \geq 5
\end{aligned}
$$

For $n=5$ :

$$
\begin{aligned}
& w t\left(u_{2} v_{2}\right)=2\left\lceil\frac{(4+n) \cdot 2+2}{3}\right]-2 ; w t\left(x_{2} v_{2}\right)=2\left\lceil\frac{(4+n) \cdot 2+2}{3}\right\rceil-1 \text {; } \\
& w t\left(u_{2} v_{3}\right)=2\left\lceil\frac{(4+n) \cdot 2+2}{3}\right\rceil ; w t\left(x_{2} v_{3}\right)=2\left\lceil\frac{(4+n) \cdot 2+2}{3}\right\rceil+1 \text {; } \\
& w t\left(x_{2} y_{2}^{1}\right)=2\left[\frac{(4+n) \cdot 2+2}{3}\right]+2 \\
& w t\left(y_{2}^{j} y_{2}^{j+1}\right)=2\left\lceil\frac{(4+n) \cdot 2+2}{3}\right\rceil+(j+2), 1 \leq j \leq n-1 .
\end{aligned}
$$

For $n=8$ :

$$
\begin{aligned}
& w t\left(u_{2} v_{2}\right)=2\left[\frac{(4+n) \cdot 2+2}{3}\right]-3 ; w t\left(x_{2} v_{2}\right)=2\left[\frac{(4+n) \cdot 2+2}{3}\right]-2 \text {; } \\
& w t\left(u_{2} v_{3}\right)=2\left\lceil\frac{(4+n) \cdot 2+2}{3}\right\rceil-1 ; w t\left(x_{2} v_{3}\right)=2\left\lceil\frac{(4+n) \cdot 2+2}{3}\right\rceil ; \\
& w t\left(x_{2} y_{2}^{1}\right)=2\left[\frac{(4+n) \cdot 2+2}{3}\right]+1 \\
& w t\left(y_{2}^{j} y_{2}^{j+1}\right)=2\left\lceil\frac{(4+n) \cdot 2+2}{3}\right\rceil+(j+1), 1 \leq j \leq n-1 .
\end{aligned}
$$

For $n \geq 11$ :

$$
\begin{array}{ll}
w t\left(u_{2} v_{2}\right)=2\left[\frac{(4+n) \cdot 2+2}{3}\right]-\left(\frac{n+1}{3}\right) ; w t\left(x_{2} v_{2}\right)=2\left\lceil\frac{(4+n) \cdot 2+2}{3}\right\rceil-\left(\frac{n+1}{3}\right)+1 ; \\
w t\left(u_{2} v_{3}\right)=2\left[\frac{(4+n) \cdot 2+2}{3}\right]-\left(\frac{n+1}{3}\right)+2 ; w t\left(x_{2} v_{3}\right)=2\left\lceil\frac{(4+n) \cdot 2+2}{3}\right\rceil-\left(\frac{n+1}{3}\right)+3 ; \\
w t\left(x_{2} y_{2}^{1}\right)=2\left[\frac{(4+n) \cdot 2+2}{3}\right]-\left(\frac{n+1}{3}\right)+4 ; \\
w t\left(y_{2}^{j} y_{2}^{j+1}\right)=2\left[\frac{(4+n) \cdot 2+2}{3}\right]+j-\left(\frac{n-11}{3}\right), 1 \leq j \leq n-1 .
\end{array}
$$


Further, for $n \geq 5$ :

$$
\begin{aligned}
& w t\left(u_{3} v_{3}\right) \quad=w t\left(u_{2} v_{2}\right)+(n+4) ; w t\left(x_{3} v_{3}\right)=w t\left(x_{2} v_{2}\right)+(n+4) ; \\
& w t\left(u_{3} v_{4}\right)=w t\left(u_{2} v_{3}\right)+(n+4) ; w t\left(x_{3} v_{4}\right)=w t\left(x_{2} v_{3}\right)+(n+4) ; \\
& w t\left(x_{3} y_{3}^{1}\right)=w t\left(x_{2} y_{2}^{1}\right)+(n+4) ; \\
& w t\left(y_{3}^{j} y_{3}^{j+1}\right)=w t\left(y_{2}^{j} y_{2}^{j+1}\right)+(n+4), 1 \leq j \leq n-1 ;
\end{aligned}
$$

It is easy to check that the largest integer used to label vertices and edges is $\left\lceil\frac{(4+n) r+2}{3}\right\rceil$ and each edge has a different weight. Hence, tes $\left(T_{r}(4, n)\right)=\left\lceil\frac{(4+n) r+2}{3}\right\rceil$.

Theorem 2.2. Let $T_{r}(4, n)$ be a tadpole chain graph with $n \equiv 2 \bmod 3, n \geq 5$, and $r \geq 1$. Then,

$$
\operatorname{tes}\left(T_{r}(4, n)\right)=\left\lceil\frac{(4+n) r+2}{3}\right\rceil .
$$

Proof. We prove the theorem by induction on $n$ and $r$. Based on Lemma 2.2, tes $\left(T_{1}(4, n)\right)=$ $\left\lceil\frac{(4+n)+2}{3}\right\rceil$. Hence, the theorem is true for $r=1$. When $n=5$, according to Lemma 2.2 we get tes $\left(T_{r}(4,5)\right)=\left\lceil\frac{9 r+2}{3}\right\rceil\left\lceil=\frac{(4+5) r+2}{3}\right\rceil$. It means that Statement (4) is true for $n=5$.

1. Statement (4) is verified by induction on $n$.

Assume that the statement is true for $n=k$, i.e.,

$$
\operatorname{tes}\left(T_{r}(4, k)\right)=\left\lceil\frac{(4+k) r+2}{3}\right\rceil .
$$

We will show that the the statement is valid for $n=k+3$ through two cases.

(a) Case 1: for $r=1$.

By means of Lemma 2.2, we get tes $\left(T_{1}(4, k+3)\right)=\left\lceil\frac{(k+3)+2}{3}\right\rceil$. Thus, the statement is true for $r=1$ and $n=k+3$.

(b) Case 2: for $r>1$.

Let $P_{k}$ be a path connected with a bridge to cycle $C_{4}$ on each block with $P_{k}=$ $v_{1} e_{1} v_{2} e_{2} v_{3} \ldots v_{k-1} e_{k-1} v_{k}$. We can get path $P_{k+3}$ to form tadpole $T(4, k+3)$ on each block by subdividing edge $e_{k-1}$ into three edges by adding three new vertices $x_{1}, x_{2}, x_{3}$ [4]. Based on assumption (5), there exists an edge irregular total $k^{\prime}$-labeling $f$ on $r$ blocks with $k^{\prime}=\left\lceil\frac{(4+k) r+2}{3}\right\rceil$. We define labels for $v_{k-1}$ and $v_{k}$ on $r$-blocks as follows: $f\left(v_{k-1}^{r}\right)=k^{\prime}-1=\left\lceil\frac{(4+k) r+2}{3}\right\rceil-1$ and $f\left(v_{k}^{r}\right)=k^{\prime}=\left\lceil\frac{(4+k) r+2}{3}\right\rceil$ with $r>1$. We construct optimal labeling on $T_{r}(4, k+3)$ on $r$-blocks by procedures as follows:

i. construct labels of vertices $f\left(x_{1}^{r}\right)=k^{\prime}, f\left(x_{2}^{r}\right)=k^{\prime}+r, f\left(x_{3}^{r}\right)=k^{\prime}+r$;

ii. construct labels of edges $f\left(v_{k-1}^{r} x_{1}^{r}\right)=k^{\prime}+r, f\left(x_{1}^{r} x_{2}^{r}\right)=k^{\prime}+r, f\left(x_{2}^{r} x_{3}^{r}\right)=k^{\prime}+$ $r, f\left(x_{3}^{r} v_{k}^{r}\right)=k^{\prime}+r$. 
By using total labeling $f$ and the above procedures, we obtain distinct weights of edges on $T_{r}(4, k+3)$. Furthermore, the largest integer for labels of vertices and edges on $T_{r}(4, k+3)$ is $k^{\prime}+r=\left\lceil\frac{(4+k) r+2}{3}\right\rceil+r=\left\lceil\frac{(4+k+3) r+2}{3}\right\rceil$. Thus, Statement (4) is true for $n=k+3$.

2. Statement (4) is proved by induction on $r$.

Assume that the theorem holds for $r$, i.e. $\operatorname{tes}\left(T_{r}(4, n)\right)=\left\lceil\frac{(4+n) r+2}{3}\right\rceil$. We will verify tes $\left(T_{r+1}(4, n)\right)$. Tadphole chain graph $T_{r+1}(4, n)$ consists of $(r+1)$ blocks, i.e., $B_{1}, B_{2}, \ldots, B_{r}$, $B_{r+1}$. By using the assumption, we have an edge irregular total $k$-labeling $f$ on $r$ blocks $B_{1}, B_{2}, \ldots, B_{r}$, with $k=\left\lceil\frac{(4+n) r+2}{3}\right\rceil$. We will show the upper bound of tes $\left(T_{r+1}(4, n)\right)$ by constructing procedures as given in Theorem 2.1 to label vertices and edges on block $B_{r+1}$ :

(a) label for each vertex on block $B_{r+1}$ is obtained by adding an integer $\frac{4+n}{3}$ to label of coresponding vertex on block $B_{r}$;

(b) label for each edge on block $B_{r+1}$ is also obtained by adding an integer $\frac{4+n}{3}$ to label of coresponding edge on block $B_{r}$.

By means of the procedures, the weight of each edge on block $B_{r+1}$ is obtained by adding a number $4+n$ to the weight of coresponding edge on block $B_{r}$. Since we have different edge weights of on block $B_{r}$ under labeling $f$, we also obtain different weights of edges on block $B_{r+1}$. Since the largest integer for labels of vertices and edges of $T_{r}(4, n)$ is $\left[\frac{(4+n) r+2}{3}\right]$, as a consequence we get the largest integer for the labels on block $B_{r+1}$ is $\left\lceil\frac{(4+n) r+2}{3}\right\rceil+\left\lceil\frac{4+n}{3}\right\rceil=$ $\left\lceil\frac{(4+n)(r+1)+2}{3}\right\rceil$ which shows the theorem holds for $r+1$. Thus, tes $\left(T_{r}(4, n)\right)=\left\lceil\frac{(4+n) r+2}{3}\right\rceil$ holds for any natural number $r$ and $n \equiv 0 \bmod 3$.

In Figure 2, we give an illustration of an edge irregular total 17-labeling of $T_{4}(4,8)$ such that $\operatorname{tes}\left(T_{4}(4,8)\right)=17$.

Further, the results of tes of $\left(T_{r}(5, n)\right)$ are presented in Lemma 2.3, Lemma 2.4, Lemma 2.5, and Theorem 2.3. 
Computing total edge irregularity strength of ... $\quad \mid \quad$ I. Rosyida and D. Indriati

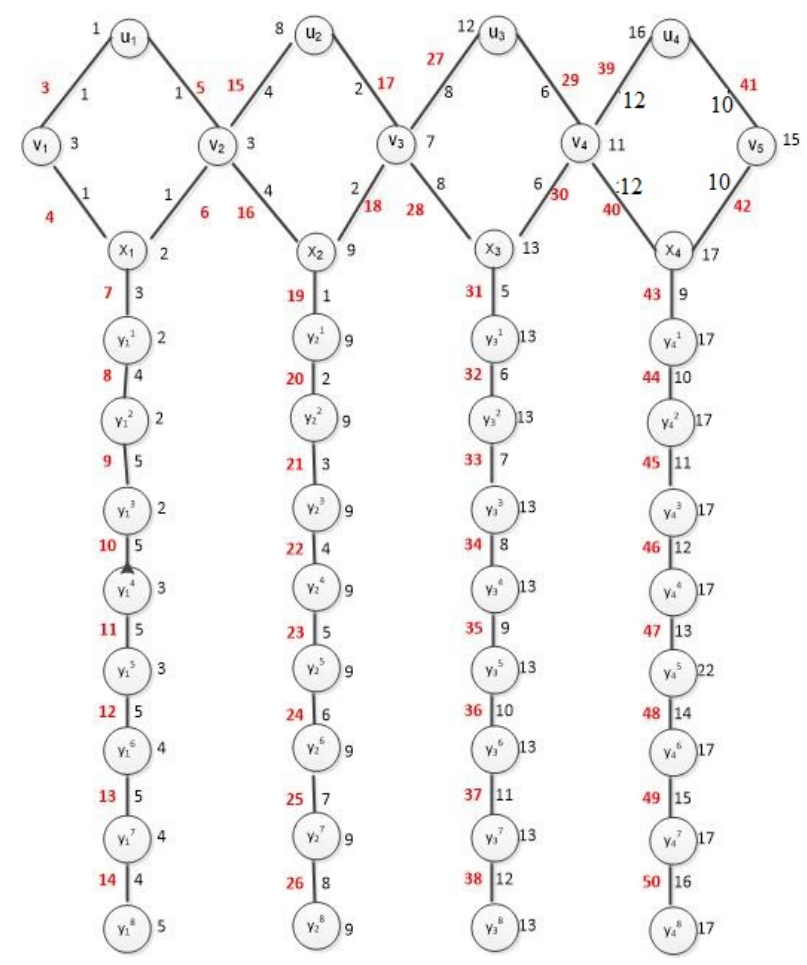

Figure 2. Edge irregular total 17-labeling of $T_{4}(4,8)$

Lemma 2.3. Let $\left(T_{r}(5,1)\right)$ be a tadpole chain graph of length $r$ with $r \geq 1$. Then,

$$
\operatorname{tes}\left(T_{r}(5,1)\right)=\left\lceil\frac{6 r+2}{3}\right\rceil
$$

Proof. Tadpole chain graph $T_{r}(5,1)$ consists of $r$ blocks $B_{1}, B_{2}, \ldots, B_{r}$ with 6 edges on each block. Let $u_{i}^{1}, u_{i}^{2}, v_{i}, v_{i+1}, x_{i}$ be vertices of cycle $C_{5}$ on each block $B_{i}$ where $v_{i+1}$ is a cut vertex between two blocks $B_{i}$ and $B_{i+1}$. Let $y_{i}^{1}$ be vertices of path graph $P_{1}$ connected with a bridge to vertices $x_{i}$. The lower bound for tes $T_{r}(5,1)$ is as follows:

$$
\left\lceil\frac{6 r+2}{3}\right\rceil \leq \operatorname{tes}\left(T_{r}(5,1)\right) \leq 6 r
$$

Further, we investigate upper bound tes $\left(T_{r}(5,1)\right) \leq\left\lceil\frac{6 r+2}{3}\right\rceil$ by constructing a total $k$-labeling $f: V \cup E \rightarrow\{1,2, \ldots, k\}$ and show that the vertex and labels are at most $k=\left\lceil\frac{6 r+2}{3}\right\rceil$ and each edge has a diverse weight under labeling $f$. Let us consider two cases.

Case 1. For $r=1,2$.

Labels of vertices are constructed in the following way: 


$$
\begin{aligned}
& f\left(u_{1}^{1}\right)=f\left(u_{1}^{2}\right)=1 ; f\left(u_{2}^{1}\right)=f\left(u_{2}^{2}\right)=\left\lceil\frac{12+2}{3}\right\rceil-1=4 ; \\
& f\left(v_{1}\right)=f\left(v_{2}\right)=1 ; f\left(v_{3}\right)=2.3-4=2 ; \\
& f\left(x_{1}\right)=2 ; f\left(x_{2}\right)=\left\lceil\frac{12+2}{3}\right\rceil=5 ; f\left(y_{i}^{1}\right)=\left\lceil\frac{6 i+2}{3}\right\rceil ; i=1,2 ;
\end{aligned}
$$

Whereas, labels of edges are constructed as follows:

$$
\begin{aligned}
& f\left(u_{1}^{1} u_{1}^{2}\right)=5 ; f\left(u_{1}^{1} v_{1}\right)=1 ; f\left(u_{1}^{2} v_{2}\right)=3 ; \\
& f\left(u_{2}^{1} u_{2}^{2}\right)=\left\lceil\frac{12+2}{3}\right\rceil=5 ; f\left(u_{2}^{1} v_{2}\right)=\left\lceil\frac{12+2}{3}\right\rceil-1=4 ; f\left(u_{2}^{2} v_{3}\right)=\left\lceil\frac{12+2}{3}\right\rceil=5 ; \\
& f\left(x_{1} v_{1}\right)=1 ; f\left(x_{1} v_{2}\right)=3 ; f\left(y_{1}^{1} x_{1}\right)=\left\lceil\frac{6+2}{3}\right\rceil=3 ; \\
& f\left(x_{2} v_{2}\right)=\left\lceil\frac{12+2}{3}\right\rceil-1=4 ; f\left(x_{2} v_{3}\right)=\left\lceil\frac{12+2}{3}\right\rceil=5 ; f\left(y_{2}^{1} x_{2}\right)=\left\lceil\frac{12+2}{3}\right\rceil-1=4 .
\end{aligned}
$$

We get the weights of edges under labeling $f$ as follows:

$$
\begin{aligned}
& w t\left(u_{1}^{1} u_{1}^{2}\right)=7 ; w t\left(u_{1}^{1} v_{1}\right)=3 ; w t\left(u_{1}^{2} v_{2}\right)=5 ; \\
& w t\left(u_{2}^{1} u_{2}^{2}\right)=3\left\lceil\frac{12+2}{3}\right\rceil-2=13 ; w t\left(u_{2}^{1} v_{2}\right)=2\left\lceil\frac{12+2}{3}\right\rceil-1=9 ; w t\left(u_{2}^{2} v_{3}\right)=2\left\lceil\frac{12+2}{3}\right\rceil+1=11 ; \\
& w t\left(x_{1} v_{1}\right)=4 ; w t\left(x_{1} v_{2}\right)=6 ; w t\left(y_{1}^{1} x_{1}\right)=2\left\lceil\frac{6+2}{3}\right\rceil+2=8 ; \\
& w t\left(x_{2} v_{2}\right)=2\left\lceil\frac{12+2}{3}\right\rceil=10 ; w t\left(x_{2} v_{3}\right)=2\left\lceil\frac{12+2}{3}\right\rceil+2=12 ; w t\left(y_{2}^{1} x_{2}\right)=3\left\lceil\frac{12+2}{3}\right\rceil-1=14 .
\end{aligned}
$$

It is easy to see that $f$ is an edge irregular total $k$-labeling that satisfies the required properties, i.e. the largest label of vertices and edges is $\left\lceil\frac{6 r+2}{3}\right\rceil$ and each edge has a different weight. Thus, $\operatorname{tes}\left(T_{r}(5,1)\right)=\left\lceil\frac{6 r+2}{3}\right\rceil$.

Case 2. For $r \geq 3$.

We construct vertex and edge labels as described below:

$$
\begin{aligned}
& f\left(u_{r}^{1}\right)=f\left(u_{r}^{2}\right)=2 r ; f\left(v_{r}\right)=2 r-4 ; f\left(v_{r+1}\right)=2 r-2 ; f\left(x_{r}\right)=\left\lceil\frac{6 r+2}{3}\right\rceil ; f\left(y_{r}^{1}\right)=\left\lceil\frac{6 r+2}{3}\right\rceil . \\
& f\left(u_{r}^{1} u_{r}^{2}\right)=\left\lceil\frac{6 r+2}{3}\right\rceil ; f\left(u_{r}^{1} v_{r}\right)=f\left(u_{r}^{2} v_{r+1}\right)=\left\lceil\frac{6 r+2}{3}\right\rceil ; \\
& f\left(x_{r} v_{r}\right)=f\left(x_{r} v_{r+1}\right)=\left\lceil\frac{6 r+2}{3}\right\rceil ; f\left(y_{r}^{1} x_{r}\right)=\left\lceil\frac{6 r+2}{3}\right\rceil-1 .
\end{aligned}
$$

Under labeling $f$, we obtain the weights of edges as follows:

$$
\begin{aligned}
& w t\left(u_{r}^{1} u_{r}^{2}\right)=4 r+\left\lceil\frac{6 r+2}{3}\right\rceil ; w t\left(u_{r}^{1} v_{r}\right)=4 r+\left\lceil\frac{6 r+2}{3}\right\rceil-4 ; w t\left(u_{r}^{2} v_{r+1}\right)=4 r+\left\lceil\frac{6 r+2}{3}\right\rceil-2 ; \\
& w t\left(x_{r} v_{r}\right)=2 r+2\left\lceil\frac{6 r+2}{3}\right\rceil-4 ; w t\left(x_{r} v_{r+1}\right)=2 r+2\left\lceil\frac{6 r+2}{3}\right\rceil-2 ; w t\left(y_{r}^{1} x_{r}\right)=3\left\lceil\frac{6 r+2}{3}\right\rceil-1 .
\end{aligned}
$$

It is shown that the largest integer of vertex and edge labels is $\left\lceil\frac{6 r+2}{3}\right\rceil$ and the edge weights are all different. It completes the proof of upper bound of $\operatorname{tes}\left(T_{r}(5,1)\right)$. Thus, tes $\left(T_{r}(5,1)\right)=$ $\left\lceil\frac{6 r+2}{3}\right\rceil$.

Lemma 2.4. Given a tadpole chain graph $\left(T_{r}(5,4)\right)$ of length $r$ with $r \geq 1$. Then, tes $\left(T_{r}(5,4)\right)=$ $\left\lceil\frac{9 r+2}{3}\right\rceil$ for $r \geq 1$.

Proof. Let $u_{i}^{1}, u_{i}^{2}, v_{i}, v_{i+1}, x_{i}$ be vertices of cycle $C_{5}$ on each block $B_{i}$ where $v_{i+1}$ is a cut vertex of two blocks $B_{i}$ and $B_{i+1}$. Let $y_{i}^{1}, y_{i}^{2}, y_{i}^{3}, y_{i}^{4}$ be vertices of the path graph $P_{4}$ connected with a bridge to vertices $x_{i}$. The lower bound of tes $\left(T_{r}(5,4)\right)$ is as follows:

$$
\left\lceil\frac{9 r+2}{3}\right\rceil \leq \operatorname{tes}\left(T_{r}(5,4)\right) \leq 9 r .
$$


To verify the upper bound of tes $\left(T_{r}(5,4)\right)$, we construct a total $k$-labeling $f: V \cup E \rightarrow$ $\{1,2, \ldots, k\}$ with $k=\left\lceil\frac{9 r+2}{3}\right\rceil$ and show that the largest integer of vertex and edge labels is $k$ and all edges has a distinct weights. Let us consider two cases.

Case 1. For $r=1,2$.

Vertex and edge labels are described below:

$$
\begin{aligned}
& f\left(u_{1}^{1}\right)=f\left(u_{1}^{2}\right)=1 ; f\left(u_{2}^{1}\right)=f\left(u_{2}^{2}\right)=\left\lceil\frac{9.2+2}{3}\right\rceil-1=6 ; \\
& f\left(v_{1}\right)=f\left(v_{2}\right)=1 ; f\left(v_{3}\right)=2 ; \\
& f\left(x_{1}\right)=1 ; f\left(x_{2}\right)=\left\lceil\frac{9.2+2}{3}\right\rceil=7 ; \\
& f\left(y_{1}^{2 i-1}\right)=i+2 ; f\left(y_{2}^{2 i}\right)=i+1, i=1,2 . \\
& f\left(u_{1}^{1} u_{1}^{2}\right)=5 ; f\left(u_{1}^{1} v_{1}\right)=1 ; f\left(u_{1}^{2} v_{2}\right)=3 ; f\left(x_{1} v_{1}\right)=2 ; f\left(x_{1} v_{2}\right)=4 ; \\
& f\left(y_{1}^{1} x_{1}\right)=f\left(y_{1}^{1} y_{1}^{2}\right)=f\left(y_{1}^{2} y_{1}^{3}\right)=f\left(y_{1}^{3} y_{1}^{4}\right)=\left\lceil\frac{9+2}{3}\right\rceil=4 ; \\
& f\left(u_{2}^{1} u_{2}^{2}\right)=\left\lceil\frac{9.2+2}{3}\right\rceil-3=4 ; \\
& f\left(u_{2}^{1} v_{2}\right)=\left\lceil\frac{9.2+2}{3}\right\rceil-2=5 ; f\left(u_{2}^{2} v_{3}\right)=\left\lceil\frac{9.2+2}{3}\right\rceil-1=6 ; \\
& f\left(x_{2} v_{2}\right)=\left\lceil\frac{9.2+2}{3}\right\rceil-2=5 ; f\left(x_{2} v_{3}\right)=\left\lceil\frac{9.2+2}{3}\right\rceil-1=6 ; \\
& f\left(y_{2}^{1} x_{2}\right)=\left\lceil\frac{9.2+2}{3}\right\rceil-4=3 ; \\
& f\left(y_{2}^{j} y_{2}^{j+1}\right)=\left\lceil\frac{9.2+2}{3}\right\rceil-4+j ; j=1,2,3 .
\end{aligned}
$$

We get the weights of edges in the following:

$$
\begin{aligned}
& w t\left(u_{1}^{1} u_{1}^{2}\right)=7 ; w t\left(u_{2}^{1} u_{2}^{2}\right)=3\left\lceil\frac{9.2+2}{3}\right\rceil-5=16 \\
& w t\left(u_{i}^{1} v_{i}\right)=9 i-6 ; w t\left(u_{i}^{1} v_{i+1}\right)=9 i-4, i=1,2 \\
& w t\left(x_{i} v_{i}\right)=9 i-5 ; w t\left(x_{i} v_{i+1}\right)=9 i-3, i=1,2 \\
& w t\left(y_{i}^{1} x_{i}\right)=9 i-1 ; w t\left(y_{i}^{j} y_{i}^{j+1}\right)=9 i+(j-1), i=1,2,3 .
\end{aligned}
$$

So the edge weights of $T_{r}(5,4)$ under labeling $f$ are different and the largest label used is $k=$ $\left\lceil\frac{9 r+2}{3}\right\rceil$. It shows that the upper bound of tes $\left(T_{r}(5,4)\right)$ is $\left\lceil\frac{9 r+2}{3}\right\rceil$. Hence, tes $\left(T_{r}(5,4)\right)=\left\lceil\frac{9 r+2}{3}\right\rceil$.

Case 2. For $r \geq 3$.

Labels of vertices and edges are provided in the following way:

$$
\begin{aligned}
& f\left(u_{r}^{1}\right)=f\left(u_{r}^{2}\right)=3 r ; f\left(v_{r}\right)=3 r-7 ; f\left(v_{r+1}\right)=3 r-4 ; \\
& f\left(x_{r}\right)=\left\lceil\frac{9 r+2}{3}\right\rceil ; f\left(y_{r}^{i}\right)=\left\lceil\frac{9 r+2}{3}\right\rceil, i=1,2,3,4 . \\
& f\left(u_{r}^{1} u_{r}^{2}\right)=\left\lceil\frac{9 r+2}{3}\right\rceil-3 ; f\left(u_{r}^{1} v_{r}\right)=\left\lceil\frac{9 r+2}{3}\right\rceil ; f\left(u_{r}^{2} v_{r+1}\right)=\left\lceil\frac{9 r+2}{3}\right\rceil-1 ; \\
& f\left(x_{r} v_{r}\right)=\left\lceil\frac{9 r+2}{3}\right\rceil ; f\left(x_{r} v_{r+1}\right)=\left\lceil\frac{9 r+2}{3}\right\rceil-1 ; f\left(y_{r}^{1} x_{r}\right)=\left\lceil\frac{9 r+2}{3}\right\rceil-4 ; \\
& f\left(y_{r}^{j} y_{r}^{j+1}\right)=\left\lceil\frac{9 r+2}{3}\right\rceil-4+j, j=1,2,3 .
\end{aligned}
$$

We observe that

$$
\begin{aligned}
& w t\left(u_{r}^{1} u_{r}^{2}\right)=6 r+\left\lceil\frac{9 r+2}{3}\right\rceil-3 ; w t\left(u_{r}^{1} v_{r}\right)=6 r+\left\lceil\frac{9 r+2}{3}\right\rceil-7 \\
& w t\left(u_{r}^{2} v_{r+1}\right)=6 r+\left\lceil\frac{9 r+2}{3}\right\rceil-5 ; w t\left(x_{r} v_{r}\right)=3 r+2\left\lceil\frac{9 r+2}{3}\right\rceil-7 \\
& w t\left(x_{r} v_{r+1}\right)=3 r+2\left\lceil\frac{9 r+2}{3}\right\rceil-5 ; w t\left(y_{r}^{1} x_{r}\right)=3\left\lceil\frac{9 r+2}{3}\right\rceil^{2}-4 \\
& w t\left(y_{r}^{j} y_{r}^{j+1}\right)=3\left\lceil\frac{9 r+2}{3}\right\rceil-4+j, j=1,2,3
\end{aligned}
$$


It is shown that the largest integer of labels of vertices and edges is $\left\lceil\frac{9 r+2}{3}\right\rceil$ and the edge weights are all distinct. It completes the proof that tes $\left(T_{r}(5,4)\right)=\left\lceil\frac{9 r+2}{3}\right\rceil$.

Lemma 2.5. Let $\left(T_{r}(5, n)\right)$ be a tadpole chain graph of length $r$ which each block is a tadpole graph $T(5, n)$ with $n \equiv 1 \bmod 3$ and $r=1,2,3$. Then,

$$
\operatorname{tes}\left(T_{r}(5, n)\right)=\left\lceil\frac{(5+n) r+2}{3}\right\rceil .
$$

Proof. Let $u_{i}^{1}, u_{i}^{2}, v_{i}, v_{i+1}, x_{i}$ be vertices of cycle $C_{5}$ on block $B_{i}$ where $v_{i+1}$ is a cut vertex of two blocks $B_{i}$ and $B_{i+1}$. Let $y_{i}^{1}, y_{i}^{2}, y_{i}^{3}, \ldots, y_{i}^{n}(i=1,2, \ldots, r)$ be vertices of path graph $P_{n}$ connected with a bridge to vertices $x_{i}$. The lower bound for tes $\left(T_{r}(5, n)\right)$ is:

$$
\left\lceil\frac{(5+n) r+2}{3}\right\rceil \leq \operatorname{tes}\left(T_{r}(5, n)\right) \leq(5+n) r .
$$

To show that $k=\left\lceil\frac{(5+n) r+2}{3}\right\rceil$ is an upper bound for tes $\left(T_{r}(5, n)\right)$, we construct a total $k$ labeling $f: V \cup E \rightarrow\{1,2, \ldots, k\}$ by considering two cases.

Case 1. For $n \equiv 1 \bmod 6, n \geq 7$, and $r=1,2$.

Labels of vertices are as follows:

$$
\begin{aligned}
& f\left(u_{1}^{1}\right)=f\left(u_{1}^{2}\right)=1 ; \\
& f\left(u_{2}^{1}\right)=f\left(u_{2}^{2}\right)=\left\lceil\frac{(5+n) 2+2}{3}\right]-1(\text { for } n=7) ; \\
& f\left(u_{2}^{1}\right)=f\left(u_{2}^{2}\right)=\left\lceil\frac{(5+n) 2+2}{3}\right\rceil-\left(2+\frac{n-13}{6}\right)(\text { for } n \geq 13) ; \\
& f\left(v_{1}\right)=f\left(v_{2}\right)=1 ; f\left(v_{3}\right)=2 ; \\
& f\left(x_{1}\right)=2 ; f\left(x_{2}\right)=\left\lceil\frac{(5+n) 2+2}{3}\right\rceil(\text { for } n=7) ; \\
& f\left(x_{2}\right)=\left\lceil\frac{(5+n) 2+2}{3}\right\rceil-\left(1+\frac{n-13}{6}\right)(\text { for } n \geq 13) ; \\
& f\left(y_{1}^{1}\right)=f\left(y_{1}^{2}\right)=2 ; f\left(y_{1}^{3}\right)=f\left(y_{1}^{4}\right)=3(\text { for } n=7) ; \\
& f\left(y_{1}^{1}\right)=f\left(y_{1}^{2}\right)=\ldots=f\left(y_{1}^{\frac{5+n}{3}}-4\right)=1(\text { for } n \geq 13) ; \\
& f\left(y_{1}^{\frac{5+n}{3}-3}\right)=f\left(y_{1}^{\frac{5+n}{3}-2}\right)=2 ; f\left(y_{1}^{\frac{5+n}{3}-1}\right)=f\left(y_{1}^{\frac{5+n}{3}}\right)=3(\text { for } n \geq 13) ; \\
& f\left(y_{1}^{\frac{5+n}{3}+i}\right)=3+\left\lfloor\frac{i+1}{2}\right\rfloor\left(\text { for } 1 \leq i \leq \frac{2 n-11}{3}+1, n \geq 7\right) ; \\
& f\left(y_{1}^{n}\right)=\left\lceil\frac{(5+n)+2}{3}\right\rceil \cdot \\
& f\left(y_{2}^{i}\right)=\left\lceil\frac{(5+n) 2+2}{3}\right\rceil \text { for } i=1,2, \ldots, n, \text { and } n=7,13 ; \\
& \quad \text { For } n \geq 19: \\
& \quad f\left(y_{2}^{1}\right)=f\left(y_{2}^{2}\right)=\left\lceil\frac{(5+n) 2+2}{3}\right]-\left(\frac{n-13}{6}\right) ; \\
& \quad f\left(y_{2}^{3}\right)=f\left(y_{2}^{4}\right)=\left[\frac{(5+n) 2+2}{3}\right\rceil-\left(\frac{n-13}{6}\right)+1 ; \\
& \quad f\left(y_{2}^{\frac{5+n}{3}-5}\right)=f\left(y_{2}^{\frac{5+n}{3}-4}\right)=\ldots=f\left(y_{2}^{n}\right)=\left\lceil\frac{(5+n) 2+2}{3}\right\rceil .
\end{aligned}
$$


Moreover, labels of edges are described below:

$$
\begin{aligned}
& f\left(u_{1}^{1} u_{1}^{2}\right)=5 ; f\left(u_{1}^{1} v_{1}\right)=1 ; f\left(u_{1}^{2} v_{2}\right)=3 ; f\left(x_{1} v_{1}\right)=2 ; f\left(x_{1} v_{2}\right)=4 ; f\left(u_{2}^{1} u_{2}^{2}\right)=3 ; \\
& f\left(u_{2}^{1} v_{2}\right)=\left[\frac{(5+n) 2+2}{3}\right]-3 ; f\left(x_{2} v_{2}\right)=\left[\frac{(5+n) 2+2}{3}\right]-3 ; n=7 ; \\
& f\left(u_{2}^{2} v_{3}\right)=\left[\frac{(5+n) 2+2}{3}\right]-2 ; f\left(x_{2} v_{3}\right)=\left\lceil\frac{(5+n) 2+2}{3}\right]-2 ; n=7 \\
& f\left(y_{1}^{1} x_{1}\right)=\left[\frac{(5+n)+2}{3}\right] ; f\left(y_{1}^{i} y_{1}^{i+1}\right)=\left[\frac{(5+n)+2}{3}\right], i=1,2, \ldots, 6 ; n=7 \\
& f\left(y_{2}^{1} x_{2}\right)=2 ; f\left(y_{2}^{i} y_{2}^{i+1}\right)=i+2, i=1,2, \ldots, 6 ; n=7 . \\
& f\left(u_{2}^{1} v_{2}\right)=f\left(x_{2} v_{2}\right)=\left[\frac{(5+n) 2+2}{3}\right]-4-\frac{(n-13)}{6} ; n \geq 13 ; \\
& f\left(u_{2}^{2} v_{3}\right)=f\left(x_{2} v_{3}\right)=\left[\frac{(5+n) 2+2}{3}\right]-3-\frac{(n-13)}{6} ; n \geq 13 \\
& f\left(y_{1}^{1} x_{1}\right)=6, n \geq 13 \\
& f\left(y_{1}^{i} y_{1}^{i+1}\right)=\left[\frac{(5+n)+2}{3}\right], i=1,2, \ldots, n-1, n=13 ; \\
& \left.f\left(y_{1}^{i} y_{1}^{i+1}\right)=-\frac{(5+n)+2}{3}\right]-\left(\frac{n-13}{3}\right)+(i-1), 1 \leq i \leq\left(\frac{5+n}{3}\right)-6, n \geq 19 ; \\
& \left.f\left(y_{1}^{i} y_{1}^{i+1}\right)=-\frac{(5+n)+2}{3}\right], \frac{5+n}{3}-5 \leq i \leq n-1, n \geq 19 ; \\
& f\left(y_{2}^{1} x_{2}\right)=1 ; f\left(y_{2}^{i} y_{2}^{i+1}\right)=1,1 \leq i \leq \frac{5+n}{3}-5, n \geq 13 \\
& f\left(y_{2}^{j} y_{2}^{j+1}\right)=j-\left(\frac{n-13}{3}\right), \frac{5+n}{3}-4 \leq j \leq n-1, n \geq 13 .
\end{aligned}
$$

\section{We get the weights of edges as follows:}

$$
w t\left(u_{1}^{1} u_{1}^{2}\right)=7 ; w t\left(u_{1}^{1} v_{1}\right)=3 ; w t\left(x_{1} v_{1}\right)=4 ; w t\left(u_{1}^{2} v_{2}\right)=5 ; w t\left(x_{2} v_{2}\right)=6,(n \geq 7) .
$$

For $n=7$ :

$$
\begin{aligned}
& w t\left(u_{2}^{1} u_{2}^{2}\right)=2\left\lceil\frac{(5+n) 2+2}{3}\right\rceil+1 ; w t\left(u_{2}^{1} v_{2}\right)=2\left\lceil\frac{(5+n) 2+2}{3}\right\rceil-3 ; w t\left(u_{2}^{2} v_{3}\right)=2\left\lceil\frac{(5+n) 2+2}{3}\right\rceil-1 ; \\
& w t\left(x_{2} v_{2}\right)=2\left\lceil\frac{(5+n) 2+2}{3}\right\rceil-2 ; w t\left(x_{2} v_{3}\right)=2\left\lceil\frac{(5+n) 2+2}{3}\right\rceil ; \\
& w t\left(y_{1}^{1} x_{1}\right)=8 ; w t\left(y_{1}^{i} y_{1}^{i+1}\right)=2\left\lceil\frac{(5+n)+2}{3}\right\rceil+(i-2), i=1,2, \ldots n-1 ; \\
& w t\left(y_{2}^{i} y_{2}^{i+1}\right)=2\left\lceil\frac{(5+n) 2+2}{3}\right\rceil+(i+2), i=1,2, \ldots n-1 ;
\end{aligned}
$$

For $n \geq 13$ :

$$
\begin{aligned}
& w t\left(u_{2}^{1} u_{2}^{2}\right)=2\left\lceil\frac{(5+n) 2+2}{3}\right\rceil-\left(\frac{5+n}{3}-5\right) ; w t\left(u_{2}^{1} v_{2}\right)=2\left\lceil\frac{(5+n) 2+2}{3}\right\rceil-\left(\frac{5+n}{3}-1\right) ; \\
& w t\left(u_{2}^{2} v_{3}\right)=2\left\lceil\frac{(5+n) 2+2}{3}\right\rceil-\left(\frac{5+n}{3}-3\right) ; \\
& w t\left(x_{2} v_{2}\right)=2\left\lceil\frac{(5+n) 2+2}{3}\right\rceil-\left(\frac{5+n}{3}-2\right) ; w t\left(x_{2} v_{3}\right)=2\left\lceil\frac{(5+n) 2+2}{3}\right\rceil-\left(\frac{5+n}{3}-4\right) ;
\end{aligned}
$$




$$
\begin{aligned}
& w t\left(y_{1}^{1} x_{1}\right)=8 ; \\
& w t\left(y_{1}^{i} y_{1}^{i+1}\right)=\left\lceil\frac{(5+n)+2}{3}\right]+(i+1), 1 \leq i \leq n-1, n=13 ; \\
& w t\left(y_{1}^{i} y_{1}^{i+1}\right)=\left\lceil\frac{(5+n)+2}{3}\right\rceil+i-\left(\frac{n-13}{3}\right)+1,1 \leq i \leq n-1, n \geq 19 ; \\
& w t\left(y_{2}^{1} x_{2}\right)=2\left\lceil\frac{(5+n) 2+2}{3}\right\rceil-\left(\frac{n-13}{3}\right) ; \\
& w t\left(y_{2}^{i} y_{2}^{i+1}\right)=2\left\lceil\frac{(5+n) 2+2}{3}\right\rceil+\left(i-\frac{n-13}{3}\right), 1 \leq i \leq n-1 .
\end{aligned}
$$

We show that labels of vertices and edges are at most $\left\lceil\frac{(5+n) r+2}{3}\right\rceil$ and the edge weights are distinct. This proves the upper bound of tes $\left(T_{r}(5, n)\right)$. Thus, tes $\left(T_{r}(5, n)\right)=\left\lceil\frac{(5+n) r+2}{3}\right\rceil$.

Case 2. For $n \equiv 4 \bmod 6, n \geq 10$, and $r=1,2$.

Labels of vertices are described in the following:

$$
\begin{aligned}
& f\left(u_{1}^{1}\right)=f\left(u_{1}^{2}\right)=1 ; \\
& f\left(u_{2}^{1}\right)=f\left(u_{2}^{2}\right)=\left\lceil\frac{(5+n) 2+2}{3}\right\rceil-\left(1-\frac{n-10}{6}\right) ; n \geq 10 ; \\
& f\left(v_{1}\right)=f\left(v_{2}\right)=1 ; f\left(v_{3}\right)=2 ; \\
& f\left(x_{1}\right)=1 ; f\left(x_{2}\right)=\left[\frac{(5+n) 2+2}{3}\right\rceil-\left(\frac{n-10}{6}\right) ; n \geq 10 ; \\
& f\left(y_{1}^{1}\right)=f\left(y_{1}^{2}\right)=\ldots=f\left(y_{1}^{\frac{5+n}{3}-4}\right)=1 ; n \geq 10 ; \\
& f\left(y_{1}^{\frac{5+n}{3}-3}\right)=f\left(y_{1}^{\frac{5+n}{3}-2}\right)=2 ; f\left(y_{1}^{\frac{5+n}{3}-1}\right)=f\left(y_{1}^{\frac{5+n}{3}}\right)=3 ; n \geq 10 ; \\
& f\left(y_{1}^{\frac{5+n}{3}}+i\right)=3+\left\lfloor\frac{i+1}{2}\right\rfloor\left(\text { for } 1 \leq i \leq \frac{2 n-11}{3}+1\right) ; n \geq 10 ; \\
& f\left(y_{1}^{n}\right)=k_{1} ; n \geq 10 ; \\
& f\left(y_{2}^{1}\right)=k_{2}-\left(\frac{n-10}{6}\right) ; n \geq 10 ; \\
& f\left(y_{2}^{i}\right)=\left\lceil\frac{(5+n) 2+2}{3}\right\rceil, 2 \leq i \leq n, n=10 ; \\
& f\left(y_{2}^{i}\right)=\left\lceil\frac{(5+n) 2+2}{3}\right\rceil-\left(\frac{n-10}{6}\right)+\left\lfloor\frac{i}{2}\right\rfloor, 2 \leq i \leq \frac{5+n}{3}-6, \text { for } n \geq 22 ; \\
& f\left(y_{2}^{j}\right)=f\left(y_{2}^{j+1}\right)=\left\lceil\frac{(5+n) 2+2}{3}\right\rceil, \frac{5+n}{3}-5 \leq j \leq n, \text { for } n \geq 16 .
\end{aligned}
$$

Furthermore, we provide labels of edges as follows:

$$
\begin{aligned}
& f\left(u_{1}^{1} u_{1}^{2}\right)=5 ; f\left(u_{1}^{1} v_{1}\right)=1 ; f\left(u_{1}^{2} v_{2}\right)=3 ; f\left(x_{1} v_{1}\right)=2 ; f\left(x_{1} v_{2}\right)=4 ; f\left(u_{2}^{1} u_{2}^{2}\right)=2 ; \\
& f\left(u_{2}^{1} v_{2}\right)=f\left(x_{2} v_{2}\right)=\left[\frac{(5+n) 2+2}{3}\right]-4-\frac{(n-10)}{6} ; n \geq 10 ; \\
& f\left(u_{2}^{2} v_{3}\right)=f\left(x_{2} v_{3}\right)=\left\lceil\frac{(5+n) 2+2}{3}\right]-3-\frac{(n-10)}{6} ; n \geq 10 \\
& f\left(y_{1}^{1} x_{1}\right)=6 ; \\
& f\left(y_{1}^{i} y_{1}^{i+1}\right)=\left[\frac{(5+n)+2}{3}\right], 1 \leq i \leq n-1, n=10 ; \\
& \left.f\left(y_{1}^{i} y_{1}^{i+1}\right)=-\frac{(5+n)+2}{3}\right]-\left(\frac{n-10}{3}\right)+i, 1 \leq i \leq \frac{5+n}{3}-6, n \geq 16 ; \\
& \left.f\left(y_{1}^{i} y_{1}^{i+1}\right)=-\frac{(5+n)+2}{3}\right\rceil, \frac{5+n}{3}-5 \leq i \leq n-1, n \geq 16
\end{aligned}
$$




$$
\begin{aligned}
& f\left(y_{2}^{1} x_{2}\right)=1 \\
& f\left(y_{2}^{j} y_{2}^{j+1}\right)=j+1, \text { for } 1 \leq j \leq n-1, n=10 \\
& f\left(y_{2}^{i} y_{2}^{i+1}\right)=1,1 \leq i \leq \frac{5+n}{3}-5, n \geq 16 \\
& f\left(y_{2}^{j} y_{2}^{j+1}\right)=j-\left(\frac{n-10}{3}\right)+1, \frac{5+n}{3}-4 \leq j \leq n-1, n \geq 16 .
\end{aligned}
$$

We derive:

$$
\begin{aligned}
& w t\left(u_{1}^{1} u_{1}^{2}\right)=7 ; w t\left(u_{1}^{1} v_{1}\right)=3 ; w t\left(x_{1} v_{1}\right)=4 ; w t\left(u_{1}^{2} v_{2}\right)=5 ; w t\left(x_{1} v_{2}\right)=6 . \\
& w t\left(u_{2}^{1} u_{2}^{2}\right)=2\left\lceil\frac{(5+n) 2+2}{3}\right]-\left(\frac{n-10}{3}\right) ; w t\left(u_{2}^{1} v_{2}\right)=2\left\lceil\frac{(5+n) 2+2}{3}\right\rceil-\left(\frac{n-10}{3}\right)-4 ; n \geq 10 ; \\
& w t\left(u_{2}^{2} v_{3}\right)=2\left\lceil\frac{(5+n) 2+2}{3}\right]-\left(\frac{n-10}{3}\right)-2 ; n \geq 10 ; \\
& w t\left(x_{2} v_{2}\right)=2\left[\frac{(5+n) 2+2}{3}\right\rceil-\left(\frac{n-10}{3}\right)-3 ; w t\left(x_{2} v_{3}\right)=2\left\lceil\frac{(5+n) 2+2}{3}\right\rceil-\left(\frac{n-10}{3}\right)-1 ; n \geq 10 . \\
& w t\left(y_{1}^{1} x_{1}\right)=8 ; \\
& w t\left(y_{1}^{i} y_{1}^{i+1}\right)=8+i, \text { for } 1 \leq i \leq n-1, n \geq 10 ; \\
& w t\left(y_{2}^{1} x_{2}\right)=2\left\lceil\frac{(5+n) 2+2}{3}\right\rceil-\left(\frac{n-10}{3}\right)+1 ; n \geq 10 ; \\
& w t\left(y_{2}^{i} y_{2}^{i+1}\right)=2\left\lceil\frac{(5+n) 2+2}{3}\right]+(i+1), \text { for } 1 \leq i \leq n-1, n=10 ; \\
& w t\left(y_{2}^{i} y_{2}^{i+1}\right)=2\left\lceil\frac{(5+n) 2+2}{3}\right\rceil+\left(i-\frac{n-10}{3}+1\right), \text { for } 1 \leq i \leq n-1, n \geq 16 .
\end{aligned}
$$

It is clear that all labels of vertices and edges are at most $\left\lceil\frac{(5+n) r+2}{3}\right\rceil$ and each edge has a different weight. Thus, tes $\left(T_{r}(5, n)\right)=\left\lceil\frac{(5+n) r+2}{3}\right\rceil$.

Case 3. For $r=3, n \equiv 1 \bmod 3$, and $n \geq 7$.

We provide Labels of vertices and edges as follows:

$$
\begin{aligned}
& f\left(u_{3}^{1}\right)=f\left(u_{3}^{2}\right)=\left\lceil\frac{(5+n) 3+2}{3}\right\rceil-1 ; f\left(v_{3}\right)=2 ; f\left(v_{4}\right)=2+\left\lceil\frac{(5+n)}{3}\right\rceil ; n \geq 7 ; \\
& f\left(x_{3}\right)=\left\lceil\frac{(5+n) 3+2}{3}\right\rceil ; f\left(y_{3}^{i}\right)=\left\lceil\frac{(5+n) 3+2}{3}\right\rceil, \text { for } i=1,2, \ldots, n ; n \geq 7 ; \\
& f\left(u_{3}^{1} u_{3}^{2}\right)=7 ; f\left(u_{3}^{1} v_{3}\right)=\left\lceil\frac{(5+n) 3+2}{3}\right\rceil ; f\left(u_{3}^{2} v_{4}\right)=\left\lceil\frac{(5+n) 3+2}{3}\right\rceil-\left(\frac{(5+n)}{3}\right)+2 ; n \geq 7 ; \\
& f\left(x_{3} v_{4}\right)=\left\lceil\frac{(5+n) 3+2}{3}\right\rceil-\left(\frac{(5+n)}{3}\right)+2 ; f\left(x_{3} v_{3}\right)=\left\lceil\frac{(5+n) 3+2}{3}\right\rceil ; n \geq 7 ; \\
& f\left(x_{3} y_{3}^{1}\right)=6 ; f\left(y_{3}^{i} y_{3}^{i+1}\right)=i+6, i=1,2, \ldots, n-1 ; n \geq 7 .
\end{aligned}
$$

We derive all edges have different weights:

$$
\begin{aligned}
& w t\left(u_{3}^{1} u_{3}^{2}\right)=2\left\lceil\frac{(5+n) 3+2}{3}\right\rceil+5 ; w t\left(u_{3}^{1} v_{3}\right)=2\left\lceil\frac{(5+n) 3+2}{3}\right\rceil+1 ; w t\left(u_{3}^{2} v_{4}\right)=2\left\lceil\frac{(5+n) 3+2}{3}\right\rceil+3 ; n \geq 7 \\
& w t\left(x_{3} v_{3}\right)=2\left\lceil\frac{(5+n) 3+2}{3}\right\rceil+2 ; w t\left(x_{3} v_{4}\right)=2\left\lceil\frac{(5+n) 3+2}{3}\right\rceil+4 ; w t\left(y_{3}^{1} x_{3}\right)=2\left\lceil\frac{(5+n) 3+2}{3}\right\rceil+6 ; n \geq 7 \\
& w t\left(y_{3}^{i} y_{3}^{i+1}\right)=2\left\lceil\frac{(5+n) 3+2}{3}\right\rceil+i+6, i=1,2, \ldots, n-1 ; n \geq 7 .
\end{aligned}
$$

Therefore, the largest integer for labels of vertices and edges is $\left\lceil\frac{(5+n) 3+2}{3}\right\rceil$ and the edge weights are diverse. Thus, tes $\left(T_{3}(5, n)\right)=\left\lceil\frac{(5+n) 3+2}{3}\right\rceil$. 
Theorem 2.3. Let $\left(T_{r}(5, n)\right)$ be a tadpole chain graph of length $r$ which each block is a tadpole graph $T(5, n)$ with $n \equiv 1 \bmod 3$ and $r \geq 1$. Then,

$$
\operatorname{tes}\left(T_{r}(5, n)\right)=\left\lceil\frac{(5+n) r+2}{3}\right\rceil .
$$

Proof. The theorem is proved by induction on $n$ and $r$. Based on Lemma 2.3, Lemma 2.4, and Lemma 2.5, tes $\left(T_{1}(5, n)\right)=\left\lceil\frac{(5+n)+2}{3}\right\rceil$ for $n \equiv 1 \bmod 3$. Hence, the theorem holds for $r=1$. For $n=1$, according to Lemma 2.3 we have $\operatorname{tes}\left(T_{r}(5,1)\right)=\left\lceil\frac{6 r+2}{3}\right\rceil=\left\lceil\frac{(5+1) r+2}{3}\right\rceil$. Therefore, Statement (6) holds for $n=1$.

1. Statement (6) is proved by induction on $n$.

Assume that the statement is true for $n=k$, i.e.,

$$
\operatorname{tes}\left(T_{r}(5, k)\right)=\left\lceil\frac{(5+k) r+2}{3}\right\rceil .
$$

We will verify that the statement is true for $n=k+3$ by considering two cases.

(a) Case 1: When $r=1$.

Based on Lemma 2.5, we have tes $\left(T_{1}(5, k+3)\right)=\left\lceil\frac{(5+k+3)+2}{3}\right\rceil$. Thus, the statement holds for $r=1$ and $n=k+3$.

(b) Case 2: When $r>1$.

The proof is analog to the proof of Case 2 in Part (1) of Theorem 2.2. By using the same procedures, Statement (6) is true for $n=k+3$.

2. Statement (6) is proved by induction on $r$.

Based on Lemma 2.3, Lemma 2.4, and Lemma 2.5, tes $\left(T_{1}(5, n)\right)=\left\lceil\frac{(5+n) 3+2}{3}\right\rceil$. Hence, the theorem is true for $r=1$ and $n \equiv 0 \bmod 3$. Assume that the theorem holds for $r$, i.e. $\operatorname{tes}\left(T_{r}(5, n)\right)=\left\lceil\frac{(5+n) r+2}{3}\right\rceil$. We will show that tes $\left(T_{r+1}(5, n)\right)=\left\lceil\frac{(5+n)(r+1)+2}{3}\right\rceil$.

Tadpole chain graph $T_{r+1}(5, n)$ consists of $(r+1)$ blocks, i.e., $B_{1}, B_{2}, \ldots, B_{r}, B_{r+1}$ where each block is a tadpole graph $T(5, n)$. Based on the assumption, there exists an edge irregular total $k$-labeling $f$ on $r$ blocks $B_{1}, B_{2}, \ldots, B_{r}$, with $k=\left\lceil\frac{(5+n) r+2}{3}\right\rceil$. We construct procedures to get labels of vertices and edges on block $B_{r+1}$ as follows:

(a) Labels of vertices on block $B_{r+1}$ are obtained by adding a number $\frac{(5+n)}{3}$ to label of vertices on block $B_{r}$ under labeling $f$.

(b) Labels of edges on block $B_{r+1}$ are also obtained by adding a number $\frac{(5+n)}{3}$ to label of edges on block $B_{r}$ under labeling $f$.

By using the above procedures, we get the edge weights on block $B_{r+1}$ as follows:

$$
\begin{aligned}
& w t\left(u_{r+1}^{1} u_{r+1}^{2}\right)=w t\left(u_{r}^{1} u_{r}^{2}\right)+(5+n) ; w t\left(u_{r+1}^{1} v_{r+1}\right)=w t\left(u_{r}^{1} v_{r}\right)+(5+n) ; \\
& w t\left(u_{r+1}^{2} v_{r+2}\right)=w t\left(u_{r}^{2} v_{r+1}\right)+(5+n) ; w t\left(x_{r+1} v_{r+1}\right)=w t\left(x_{r} v_{r}\right)+(5+n) ; \\
& w t\left(x_{r+1} v_{r+2}\right)=w t\left(x_{r} v_{r+1}\right)+(5+n) ; w t\left(y_{r+1}^{1} x_{r+1}\right)=w t\left(y_{r}^{1} x_{r}\right)+(5+n) ; \\
& w t\left(y_{r+1}^{i} y_{r+1}^{i+1}\right)=w t\left(y_{r}^{i} y_{r}^{i+1}\right)+(5+n), \text { for } i=1,2, \ldots, n-1 .
\end{aligned}
$$


Since the edge weights of $T_{r}(5, n)$ are distinct under labeling $f$, we also obtain the edge weights on block $B_{r+1}$ are all distinct. Moreover,the largest integer of labels of vertices and edges of $T_{r}(5, n)$ is $\left[\frac{(5+n) r+2}{3}\right]$. As a consequence, the largest integer of labels of vertices and edges on block $B_{r+1}$ is $\left\lceil\frac{(5+n) r+2}{3}\right\rceil+\left\lceil\frac{(5+n)}{3}\right\rceil=\left\lceil\frac{(5+n)(r+1)+2}{3}\right\rceil$. Hence, tes $\left(T_{r+1}(5, n)\right)=$ $\left\lceil\frac{(5+n)(r+1)+2}{3}\right\rceil$. Thus, the theorem is true for any $r$ and $n \equiv 0 \bmod 3$. The proof is complete.

\section{Conclusions}

In this paper, we have found tes of some $n$-uniform cactus chain graphs $C\left(C_{n}^{r}\right)$ and tadpole chain graphs $T_{r}(4, n), T_{r}(5, n)$ of length $r$ with $n=0 \bmod 3$. As further research, we will determine tes of $n$-uniform cactus chain graphs $C\left(C_{n}^{r}\right)$ for $n \equiv 1 \bmod 3$ and $n \equiv 2 \bmod 3$. Also, we will investigate tvs of $n$-uniform cactus chain graphs and related chain graphs which are still in progress.

\section{References}

[1] A. Ahmad, M. Bača, Y. Bashir, and M. K. Siddiqui, Total edge irregularity strength of strong product of two paths, Ars Combin. 106 (2012), 449-459.

[2] A. Ahmad, A. Gupta, and R. Simanjuntak, Computing the edge irregularity strengths of chain graphs and the join of two graphs, Electron. J. Graph Theory Appl. 6(1) (2018), 201-207

[3] S. Alikhani, S. Jahari, M. Mehryar, and R. Hasni, Counting the number of dominating sets of cactus chains, J. Optoelectron. Adv. M. 8(9-10) (2014), 955-960.

[4] M. Bača, S. Jendrol̆, M. Miller, and J. Ryan, On irregular total labeling, Discrete Math., 307 (2007), 1378-1388.

[5] M. Bača, S. Jendroľ, K. Kathiresan, K. Muthugurupackiam, and A. Semanicová-Fenovcikova, A Survey of Irregularity Strength, Electron Notes Discrete Math. 48 (2015), 19-26.

[6] K. Borissevich and T. Došlić, Counting dominating sets in cactus chains, Filomat 29(8) (2015), 1847-1855.

[7] J. A. Gallian, A dynamic survey of graph labeling, Electron. J. Combin., 19 (2017) \#DS6.

[8] M. Imran, A. Aslam, S. Zafar, and W. Nazeer, Further results on edge irregularity strength of graphs, Indones. J. Combin. 1(2) (2017), 82-91.

[9] D. Indriati, Widodo, I. E., Wijayanti, and K. A. Sugeng, On the total edge irregularity strength of generalized helm, AKCE Int. J. Graphs Comb. 10(2) (2013), 147-155. 
[10] D. Indriati, Widodo, I. E. Wijayanti, K. A. Sugeng, and M. Bača, On total edge irregularity strength of generalized web graphs and related graphs, Math. Comput. Sci. 9 (2015), 161167.

[11] J. Ivančo and S. Jendrol̆, Total edge irregularity strength of trees, Discuss. Math. Graph Theory 26 (2006), 449-456.

[12] S. Jendroľ, J. Miškuf, and R. Soták, Total edge irregularity strength of complete graphs and complete bipartite graphs, Discrete Math. 310(3) (2010), 400-407.

[13] P. Jeyanthi and A. Sudha, Total edge irregularity strength ofsome families of graphs, Utilitas Math., 109 (2018), 139-153.

[14] J. Miškuf and S. Jendrol, On total edge irregularity strength of the grids, Tatra Mt. Math. Publ. 36 (2007), 147-151.

[15] Nurdin, A. N. M. Salman, and E. T. Baskoro, The total edge irregularity strengths of the corona product of paths with some graphs, J. Combin. Math. Combin. Comput. 65 (2008), 163-175.

[16] I. Rosyida and D. Indriati, On total edge irregularity strength of some cactus chain graphs with pendant vertices, J. Phys. Conf. Ser. J 1211(012016) (2019), 1-9.

[17] A. Sadeghieh, S. Alikhani, N. Ghanbari, and A. J. M. Khalaf, Hosoya polynomial of some cactus chains, Cogent Math. 4 (1) (2017), 1-7.

[18] M. K. Siddiqui, On edge irregularity strength of subdivision of star $S_{n}$, Int. J. of Math and Soft Comput. 2(1) (2012), 75-82. 